\title{
Numerical Study for Time Delay Multistrain Tuberculosis Model of Fractional Order
}

\author{
Nasser Hassan Sweilam, ${ }^{1}$ Seham Mahyoub Al-Mekhlafi, ${ }^{2}$ \\ and Taghreed Abdul Rahman Assiri ${ }^{3}$ \\ ${ }^{1}$ Department of Mathematics, Faculty of Science, Cairo University, Giza, Egypt \\ ${ }^{2}$ Department of Mathematics, Faculty of Education, Sana'a University, Sana'a, Yemen \\ ${ }^{3}$ Department of Mathematics, Faculty of Science, Umm-Alqura University, Makkah, Saudi Arabia \\ Correspondence should be addressed to Nasser Hassan Sweilam; nsweilam@sci.cu.edu.eg
}

Received 18 February 2017; Revised 13 April 2017; Accepted 14 May 2017; Published 31 July 2017

Academic Editor: Fathalla A. Rihan

Copyright (C) 2017 Nasser Hassan Sweilam et al. This is an open access article distributed under the Creative Commons Attribution License, which permits unrestricted use, distribution, and reproduction in any medium, provided the original work is properly cited.

\begin{abstract}
A novel mathematical fractional model of multistrain tuberculosis with time delay memory is presented. The proposed model is governed by a system of fractional delay differential equations, where the fractional derivative is defined in the sense of the Grünwald-Letinkov definition. Modified parameters are introduced to account for the fractional order. The stability of the equilibrium points is investigated for any time delay. Nonstandard finite deference method is proposed to solve the resulting system of fractional-order delay differential equations. Numerical simulations show that nonstandard finite difference method can be applied to solve such fractional delay differential equations simply and effectively.
\end{abstract}

\section{Introduction}

It is known that tuberculosis (TB) is one of the most important infectious diseases and is considered as the second largest cause of mortality by infectious diseases and a challenging disease to control [1]. Time delays required to treatment of active TB present a major obstacle to the control of a TB epidemic [2]; it worsens the disease, increases the risk of death, and enhances tuberculosis transmission to the community $[3,4]$. Both patient and the health system may be responsible for the treatment delay [3]. On the other hand, mathematical models are quite important and efficient tool to describe and investigate TB diseases; see [5-9]. In [10], Silva et al. presented TB model with time-delay memory. Herein, we consider a general model of multistrain TB diseases with time-delay memory. A discrete time delay is incorporated, in the variables of active TB infection of two and three strains, to represent the required time to commencement of treatment and diagnosis [11].
The multistrain TB model incorporates three strains: extensively drug-resistant (XDR), emerging multidrug resistant (MDR), and drug sensitive, and has been developed by Arino and Soliman [12] in 2015. Several factors of spreading TB such as the exogenous reinfection, the fast infection, and secondary infection are included in this model. Sweilam et al. introduced some numerical studies for this model in [13-16].

Fractional differential equations have been the focus of many studies due to their frequent appearance in various sciences [13-20]. The general theory of differential equations with delays (DDEs) is widely developed and discussed in the literature [21-25]. Delayed fractional differential equations (DFDEs) are also used to describe dynamical systems [2628]. Recently, DFDEs begin to raise the attention of many researchers [29-33]. Relatable and efficient numerical techniques for DFDEs are very necessary and important [34]. Nonstandard finite difference method (NSFDM) was firstly proposed by Mickens [35] in 1980s to solve numerically the ordinary differential equations (ODEs) and partial differential 
TABLE 1: Interpretation of the variable states of system (1).

\begin{tabular}{ll}
\hline Variable & Interpretation \\
\hline$S(t)$ & Individuals have never encountered TB. \\
$L_{s}(t)$ & The individuals infected with drug-sensitive TB but not infectious. \\
$L_{m}(t)$ & Infected with MDR-TB but not infectious. \\
$L_{x}(t)$ & Infected with XDR-TB but not infectious. \\
$I_{s}(t)$ & Able to infect others with drug sensitive strain. \\
$I_{m}(t)$ & Able to infect others with MDR strain. \\
$I_{x}(t)$ & Able to infect others with XDR strain. \\
$R(t)$ & Recovered by getting a successful treatment. \\
$N(t)$ & The variable of population size. \\
& $N(t)=S(t)+L_{s}(t)+L_{m}(t)+L_{x}(t)+I_{s}(t)+I_{m}(t)+I_{x}(t)+R(t)$. \\
\hline
\end{tabular}

TABLE 2: All adapted parameters and their interpretation of system (1).

\begin{tabular}{ll}
\hline Parameter & Interpretation \\
\hline$d^{\alpha}$ & Natural death rate \\
$b^{\alpha}$ & Birth rate \\
$\lambda_{j}^{\alpha}$ & Rate of infected individuals move to $L_{j}$ with strain $j \in\{s, m, x\}$ \\
$1-\lambda_{j}^{\alpha}$ & Rate of newly infected individuals progressing to active TB with strain $j \in\{s, m, x\}$ \\
$\beta_{j}^{\alpha}$ & Transmission coefficient with strain $j \in\{s, m, x\}$ \\
$\varepsilon_{j}^{\alpha}$ & Rate of endogenous reactivation of $L_{j}$ \\
$\gamma_{j}^{\alpha}$ & Rate of natural recovery to the latent stage $L_{j}$ \\
$\delta_{j}^{\alpha}$ & Rate of death due to TB of strain $j$ \\
$\alpha_{j 1}^{\alpha}, \alpha_{j 2}^{\alpha}$ & Rate of exogenous reinfection of $L_{j 1}$ due to contact with $I_{j 2}$ \\
$1-\sigma_{j}^{\alpha}$ & Efficiency of treatment in preventing infection with strain $j$ \\
$P_{1}^{\alpha}$ & Probability of treatment success for $L_{s}$ \\
$1-P_{1}^{\alpha}$ & Proportion of treated $L_{s}$ moved to $L_{m}$ due to incomplete treatment or lack of strict compliance in the use of drugs \\
$P_{2}^{\alpha}$ & Probability of treatment success for $I_{s}$ \\
$1-P_{2}^{\alpha}$ & Proportion of treated $I_{s}$ moved to $L_{m}$ due to incomplete treatment or lack of strict compliance in the use of drugs \\
$P_{3}^{\alpha}$ & Probability of treatment success for $I_{m}$ \\
$1-P_{3}^{\alpha}$ & proportion of treated $I_{m}$ moved to $L_{x}$ due to incomplete treatment or lack of strict compliance in the use of drugs \\
$t_{1 s}^{\alpha}$ & Rate of treatment for $L_{s}$ \\
$t_{2 j}^{\alpha}$ & Rate of treatment for $I_{j}$. Note that $t_{2 x}$ is the rate of successful treatment of $I_{x}, j \in\{x, m, s\}$ \\
\hline
\end{tabular}

equations (PDEs) with more accuracy than standard finite difference method (SFDM). It is considered as a powerful numerical scheme that preserves properties of exact solutions of the differential equation [36].

The main aim of work is to study numerically the solutions of fractional-order model of multistrain TB with time delay memory. The presence of fractional-order and time delays in the model can lead to a notable increase in the complexity of the observed behavior, and the solution continuously depends on all the previous states. An efficient numerical method, NSFDM, is used to numerically solve the fractional-order delay model. The rest of the paper is organized as follows: In Section 2, we present a fractional order model with time delay for multistrain TB. Stability of equilibrium points is presented in Section 3. NSFD for fractional-order delay differential equations is introduced in Section 4. Some numerical simulations are given in Section 5, and conclusion in Section 6. Some definitions on fractional calculus and some properties of nonstandard discretization are given in Appendix.

\section{Fractional Multistrain TB Model with Time Delay}

In this section, a multistrain TB model of fractional-order and time delay memory is presented. The population of interest is divided into eight compartments depending on their epidemiological stages as follows: susceptible $(S)$; latently infected with drug sensitive TB $\left(L_{s}\right)$; latently infected with $\operatorname{MDR}$ TB $\left(L_{m}\right)$; latently infected with XDR TB $\left(L_{x}\right)$; sensitive drug TB infectious $\left(I_{s}\right)$; MDR TB infectious $\left(I_{m}\right)$; XDR TB infectious $\left(I_{x}\right)$; recovered $R$. One biological meaning of the given parameters is given in Table 1 . One of the main assumptions of this model is that the total population $N(t)$, with $N(t)=S(t)+L_{s}(t)+L_{m}(t)+L_{x}(t)+I_{s}(t)+$ $I_{m}(t)+I_{x}(t)+R(t)$, is variable of the time. We introduce a discrete time delay in the state variables $I_{m}$ and $I_{x}$, denoted by $\tau$, that represents the time required for diagnosis and commencement of treatment of active TB infection of two and three strains. The parameters in the modified the model are described in Table 2; see [37]. The modified system of multistrain TB model of fractional-order and time delay is 


$$
\begin{aligned}
& D_{t}^{\alpha} S=b^{\alpha}-d^{\alpha} S-\beta_{s}^{\alpha} \frac{S I_{s}}{N}-\beta_{m}^{\alpha} \frac{S I_{m}}{N}-\beta_{x}^{\alpha} \frac{S I_{x}}{N}, \\
& D_{t}^{\alpha} L_{s}=\lambda_{s}^{\alpha} \beta_{s}^{\alpha} \frac{S I_{s}}{N}+\sigma_{s}^{\alpha} \lambda_{s}^{\alpha} \beta_{s}^{\alpha} \frac{R I_{s}}{N}+\gamma_{s}^{\alpha} I_{s}-\alpha_{s s}^{\alpha} \beta_{s}^{\alpha} \frac{L_{s} I_{s}}{N} \\
& -\alpha_{s m}^{\alpha} \beta_{m}^{\alpha} \frac{L_{s} I_{m}}{N}-\alpha_{s x}^{\alpha} \beta_{x}^{\alpha} \frac{L_{s} I_{x}}{N}-\left(d^{\alpha}+\varepsilon_{s}^{\alpha}+t_{1 s}^{\alpha}\right) L_{s}, \\
& D_{t}^{\alpha} L_{m}=\lambda_{m}^{\alpha} \beta_{m}^{\alpha} \frac{S I_{m}}{N}+\sigma_{m}^{\alpha} \lambda_{m}^{\alpha} \beta_{m}^{\alpha} \frac{R I_{m}}{N}+\gamma_{m}^{\alpha} I_{m} \\
& +\alpha_{s m}^{\alpha} \beta_{m}^{\alpha} \lambda_{m}^{\alpha} \frac{L_{s} I_{m}}{N}+\left(1-P_{1}^{\alpha}\right) t_{1 s}^{\alpha} L_{s}+\left(1-P_{2}^{\alpha}\right) t_{2 s}^{\alpha} I_{s} \\
& -\alpha_{m m}^{\alpha} \beta_{m}^{\alpha} \frac{L_{m} I_{m}}{N}-\alpha_{m x}^{\alpha} \beta_{x}^{\alpha} \frac{L_{m} I_{x}}{N}-\left(d^{\alpha}+\varepsilon_{m}^{\alpha}\right) L_{m}, \\
& D_{t}^{\alpha} L_{x}=\lambda_{x}^{\alpha} \beta_{x}^{\alpha} \frac{S I_{x}}{N}+\sigma_{x}^{\alpha} \lambda_{x}^{\alpha} \beta_{x}^{\alpha} \frac{R I_{x}}{N}+\gamma_{x}^{\alpha} I_{x}+\alpha_{s x}^{\alpha} \beta_{x}^{\alpha} \lambda_{x}^{\alpha} \\
& \cdot \frac{L_{s} I_{x}}{N}+\alpha_{m x}^{\alpha} \beta_{x}^{\alpha} \lambda_{x}^{\alpha} \frac{L_{m} I_{x}}{N}+\left(1-P_{3}^{\alpha}\right) t_{2 m}^{\alpha} I_{m}-\alpha_{x x}^{\alpha} \beta_{x}^{\alpha} \\
& \frac{L_{x} I_{x}}{N}-\left(d^{\alpha}+\varepsilon_{x}^{\alpha}\right) L_{x} \\
& D_{t}^{\alpha} I_{s}=\alpha_{s s}^{\alpha} \beta_{s}^{\alpha} \frac{L_{s} I_{s}}{N}+\left(1-\lambda_{s}^{\alpha}\right) \beta_{s}^{\alpha}\left(\frac{S I_{s}}{N}+\sigma_{s}^{\alpha} \frac{R I_{s}}{N}\right) \\
& +\varepsilon_{s}^{\alpha} L_{s}-\left(d^{\alpha}+\delta_{s}^{\alpha}+t_{2 s}^{\alpha}+\gamma_{s}^{\alpha}\right) I_{s}, \\
& D_{t}^{\alpha} I_{m}=\alpha_{m m}^{\alpha} \beta_{m}^{\alpha} \frac{L_{m} I_{m}}{N}+\left(1-\lambda_{m}^{\alpha}\right) \\
& \cdot \beta_{m}^{\alpha}\left(\frac{S I_{m}}{N}+\sigma_{m}^{\alpha} \frac{R I_{m}}{N}+\alpha_{s m}^{\alpha} \frac{L_{s} I_{m}}{N}\right)+\varepsilon_{m}^{\alpha} L_{m} \\
& -\left(d^{\alpha}+\delta_{m}^{\alpha}+\gamma_{m}^{\alpha}\right) I_{m}-t_{2 m}^{\alpha} I_{m}(t-\tau), \\
& D_{t}^{\alpha} I_{x}=\alpha_{x x}^{\alpha} \beta_{x}^{\alpha} \frac{L_{x} I_{x}}{N}+\left(1-\lambda_{x}^{\alpha}\right) \\
& \cdot \beta_{x}^{\alpha}\left(\frac{S I_{x}}{N}+\sigma_{x}^{\alpha} \frac{R I_{x}}{N}+\alpha_{s x}^{\alpha} \frac{L_{s} I_{x}}{N}+\alpha_{m x}^{\alpha} \frac{L_{m} I_{x}}{N}\right)+\varepsilon_{x}^{\alpha} L_{x} \\
& -\left(d^{\alpha}+\delta_{x}^{\alpha}+\gamma_{x}^{\alpha}\right) I_{x}-t_{2 x}^{\alpha} I_{x}(t-\tau), \\
& D_{t}^{\alpha} R=P_{1}^{\alpha} t_{1 s}^{\alpha} L_{s}+P_{2}^{\alpha} t_{2 s}^{\alpha} I_{s}+P_{3}^{\alpha} t_{2 m}^{\alpha} I_{m}+t_{2 x}^{\alpha} I_{x}(t-\tau) \\
& -\sigma_{s}^{\alpha} \beta_{s}^{\alpha} \frac{R I_{s}}{N}-\sigma_{m}^{\alpha} \beta_{m}^{\alpha} \frac{R I_{m}}{N}-\sigma_{x}^{\alpha} \beta_{x}^{\alpha} \frac{R I_{x}}{N}-d^{\alpha} R \text {. }
\end{aligned}
$$

The initial conditions for system $(1)$ are $S(\xi)=\theta_{1}(\xi), L_{s}(\xi)=$ $\theta_{2}(\xi), L_{m}(\xi)=\theta_{3}(\xi), L_{x}(\xi)=\theta_{4}(\xi), I_{s}(\xi)=\theta_{5}(\xi), I_{m}(\xi)=$ $\theta_{6}(\xi), I_{x}(\xi)=\theta_{7}(\xi), R(\xi)=\theta_{8}(\xi), \xi \in[-\tau, 0]$, where $\theta=\left(\theta_{1}, \theta_{2}, \ldots, \theta_{8}\right)^{T} \in C$, where $C$ is the Banach space $C\left([0, \tau], \mathbb{R}^{8}\right)$. From biological meaning, we further assume that $\theta_{i}>0$ for $i=1, \ldots, 8$. Throughout this paper, we focus on the dynamics of the solutions of (1) in the restricted region, $\Omega=\left\{\left(S, L_{s}, L_{m}, L_{x}, I_{s}, I_{m}, I_{x}, R\right) \in \mathbb{R}^{8} \mid S+L_{s}+L_{m}+L_{x}+\right.$ $\left.I_{s}+I_{m}+I_{x}+R \leq b^{\alpha} / d^{\alpha}\right\}$. We refer here to [24, 31], for local existence, uniqueness, and continuation results.

The unique solution $\left(S(t), L_{s}(t), L_{m}(t), L_{x}(t), I_{s}(t), I_{m}(t)\right.$, $\left.I_{x}(t), R(t)\right)$ of (1) with initial condition exists for all time $t \geq 0$. Consider the solutions of (1), for $\left(S, L_{s}\right.$, $\left.L_{m}, L_{x}, I_{s}, I_{m}, I_{x}, R\right) \in \Omega^{\prime}$, where $\Omega^{\prime}$ is the interior of $\Omega$, for all $\xi \in[-\tau, 0]$. Then the solutions stay in the interior of the region for all time $t \geq 0$; that is, the region is positively invariant with respect to system (1) (see, e.g., [31]). Model (1) has a disease-free equilibrium given by $E_{0}=$ $\left(b^{\alpha} / d^{\alpha}, 0,0,0,0,0,0,0\right)$; see [32].

2.1. Basic Reproduction Number. The basic reproduction number, $R_{0}$, is defined as the expected number of secondary cases produced, in a completely susceptible population, by a typical infective individual [32]. Herein, we apply the method in [32] to drive $R_{0}$. The order of the infected variables is

$$
\mathfrak{J}:=\left(L_{s}, L_{m}, L_{x}, I_{s}, I_{m}, I_{x}\right)^{\prime} .
$$

The vector representing new infections into the infected classes $F$ is given by

$$
F:=\left(\begin{array}{c}
\lambda_{s}^{\alpha} \beta_{s}^{\alpha} \frac{S I_{s}}{N}+\sigma_{s}^{\alpha} \lambda_{s}^{\alpha} \beta_{s}^{\alpha} \frac{R I_{s}}{N} \\
\lambda_{m}^{\alpha} \beta_{m}^{\alpha} \frac{S I_{m}}{N}+\sigma_{m}^{\alpha} \lambda_{m}^{\alpha} \beta_{m}^{\alpha} \frac{R I_{m}}{N} \\
\lambda_{x}^{\alpha} \beta_{x}^{\alpha} \frac{S I_{x}}{N}+\sigma_{x}^{\alpha} \lambda_{x}^{\alpha} \beta_{x}^{\alpha} \frac{R I_{x}}{N} \\
\left(1-\lambda_{s}^{\alpha}\right) \beta_{s}^{\alpha}\left(\frac{S I_{s}}{N}+\sigma_{s}^{\alpha} \frac{R I_{s}}{N}\right) \\
\left(1-\lambda_{m}^{\alpha}\right) \beta_{m}^{\alpha}\left(\frac{S I_{m}}{N}+\sigma_{m}^{\alpha} \frac{R I_{m}}{N}\right) \\
\left(1-\lambda_{x}^{\alpha}\right) \beta_{x}^{\alpha}\left(\frac{S I_{x}}{N}+\sigma_{x}^{\alpha} \frac{R I_{x}}{N}\right)
\end{array}\right) .
$$

The vector $V$ representing other flows within and out of the infected classes $\mathfrak{I}$ is given by

$$
V:=\left(\begin{array}{c}
\alpha_{s s}^{\alpha} \beta_{s}^{\alpha} \frac{L_{s} I_{s}}{N}-\alpha_{s m}^{\alpha} \beta_{m}^{\alpha} \frac{L_{s} I_{m}}{N}-\alpha_{s x}^{\alpha} \beta_{x}^{\alpha} \frac{L_{s} I_{x}}{N}+\gamma_{s}^{\alpha} I_{s}-\left(d^{\alpha}+\varepsilon_{s}^{\alpha}+t_{1 s}^{\alpha}\right) L_{s}, \\
+\gamma_{m}^{\alpha} I_{m}+\alpha_{s m}^{\alpha} \beta_{m}^{\alpha} \lambda_{m}^{\alpha} \frac{L_{s} I_{m}}{N}+\left(1-P_{1}^{\alpha}\right) t_{1 s}^{\alpha} L_{s}+\left(1-P_{2}^{\alpha}\right) t_{2 s}^{\alpha} I_{s}, \\
-\alpha_{m m}^{\alpha} \beta_{m}^{\alpha} \frac{L_{m} I_{m}}{N}-\alpha_{m x}^{\alpha} \beta_{x}^{\alpha} \frac{L_{m} I_{x}}{N}-\left(d^{\alpha}+\varepsilon_{m}^{\alpha}\right) L_{m}, \\
+\gamma_{x}^{\alpha} I_{x}+\alpha_{s x}^{\alpha} \beta_{x}^{\alpha} \lambda_{x}^{\alpha} \frac{L_{s} I_{x}}{N}+\alpha_{m x}^{\alpha} \beta_{x}^{\alpha} \lambda_{x}^{\alpha} \frac{L_{m} I_{x}}{N}+\left(1-P_{3}^{\alpha}\right) t_{2 m}^{\alpha} I_{m}-\alpha_{x x}^{\alpha} \beta_{x}^{\alpha} \frac{L_{x} I_{x}}{N}-\left(d^{\alpha}+\varepsilon_{x}^{\alpha}\right) L_{x}, \\
\alpha_{s s}^{\alpha} \beta_{s}^{\alpha} \frac{L_{s} I_{s}}{N}+\varepsilon_{s}^{\alpha} L_{s}-\left(d^{\alpha}+\delta_{s}^{\alpha}+t_{2 s}^{\alpha}+\gamma_{s}^{\alpha}\right) I_{s}, \\
\left(\alpha_{m m}^{\alpha} \beta_{m}^{\alpha} \frac{L_{m} I_{m}}{N}+\alpha_{s m}^{\alpha} \frac{L_{s} I_{m}}{N}\right)+\varepsilon_{m}^{\alpha} L_{m}-\left(d^{\alpha}+\delta_{m}^{\alpha}+\gamma_{m}^{\alpha}+t_{2 m}^{\alpha}\right) I_{m}, \\
\left(\alpha_{x x}^{\alpha} \beta_{x}^{\alpha} \frac{L_{x} I_{x}}{N}+\left(1-\lambda_{x}^{\alpha}\right) \beta_{x}^{\alpha}+\alpha_{s x}^{\alpha} \frac{L_{s} I_{x}}{N}+\alpha_{m x}^{\alpha} \frac{L_{m} I_{x}}{N}\right)+\varepsilon_{x}^{\alpha} L_{x}-\left(d^{\alpha}+\delta_{x}^{\alpha}+\gamma_{x}^{\alpha}+t_{2 x}^{\alpha}\right) I_{x},
\end{array}\right) .
$$


The matrix of new infections $F$ and the matrix of transfers between compartments $V$ are the Jacobian matrices obtained by differentiating $F$ and $V$ with respect to the infected variables $\mathfrak{I}$ and evaluating at the disease-free equilibrium. They take the form

$$
\begin{aligned}
& F:=\left(\begin{array}{ll}
0 & A \\
0 & B
\end{array}\right), \\
& V:=\left(\begin{array}{ll}
C & D \\
E & F_{2}
\end{array}\right),
\end{aligned}
$$

where

$$
\begin{aligned}
& A=\left(\begin{array}{ccc}
\lambda_{s}^{\alpha} \beta_{s}^{\alpha} & 0 & 0 \\
0 & \lambda_{m}^{\alpha} \beta_{m}^{\alpha} & 0 \\
0 & 0 & \lambda_{s}^{\alpha} \beta_{s}^{\alpha}
\end{array}\right) \\
& B=\left(\begin{array}{ccc}
\left(1-\lambda_{s}^{\alpha}\right) \beta_{s}^{\alpha} & 0 & 0 \\
0 & \left(1-\lambda_{m}^{\alpha}\right) \beta_{m}^{\alpha} & 0 \\
0 & 0 & \left(1-\lambda_{s}^{\alpha}\right) \beta_{s}^{\alpha}
\end{array}\right) \\
& C=\left(\begin{array}{ccc}
\left(d^{\alpha}+\varepsilon_{s}^{\alpha}+t_{1 s}^{\alpha}\right) & 0 & 0 \\
\left(-1+P_{1}^{\alpha}\right) t_{1 s}^{\alpha} & \left(d^{\alpha}+\varepsilon_{m}^{\alpha}\right) & 0 \\
0 & 0 & \left(d^{\alpha}+\varepsilon_{x}^{\alpha}\right)
\end{array}\right) \\
& D=\left(\begin{array}{ccc}
-\gamma_{s}^{\alpha} & 0 & 0 \\
\left(-1+P_{2}^{\alpha}\right) t_{2 s}^{\alpha} & -\gamma_{m}^{\alpha} & 0 \\
0 & \left(-1+P_{3}^{\alpha}\right) t_{2 m}^{\alpha} & -\gamma_{x}^{\alpha}
\end{array}\right), \\
& F_{2}=\left(\begin{array}{ccc}
\left(d^{\alpha}+\delta_{s}^{\alpha}+\gamma_{s}^{\alpha}+t_{2 s}^{\alpha}\right) & 0 & 0 \\
0 & \left(d^{\alpha}+\delta_{m}^{\alpha}+\gamma_{m}^{\alpha}+t_{2 m}^{\alpha}\right) & 0 \\
0 & 0 & \left(d^{\alpha}+\delta_{x}^{\alpha}+\gamma_{x}^{\alpha}+t_{2 x}^{\alpha}\right)
\end{array}\right) \text {, } \\
& E=\left(\begin{array}{ccc}
-\varepsilon_{s}^{\alpha} & 0 & 0 \\
0 & -\varepsilon_{m}^{\alpha} & 0 \\
0 & 0 & -\varepsilon_{x}^{\alpha}
\end{array}\right)
\end{aligned}
$$

Then the basic reproduction number $R_{0}$ for system (1) is the spectral radius of the next generation matrix and is given by

$$
R_{0}=\rho\left(F V^{-1}\right)=\max \left(R_{0 s}, R_{0 m}, R_{0 x}\right)
$$

where

$$
\begin{aligned}
R_{0 s} & =\frac{\beta_{s}^{\alpha}\left(\varepsilon_{s}^{\alpha}+\left(1-\lambda_{s}^{\alpha}\right)\left(d^{\alpha}+t_{1 s}^{\alpha}\right)\right)}{\left(\varepsilon_{s}^{\alpha}+d^{\alpha}+t_{1 s}^{\alpha}\right)\left(t_{2 s}^{\alpha}+\delta_{s}^{\alpha}+d^{\alpha}\right)+\gamma_{s}^{\alpha}\left(t_{1 s}^{\alpha}+d^{\alpha}\right)}, \\
R_{0 m} & =\frac{\beta_{m}^{\alpha}\left(\varepsilon_{m}^{\alpha}+\left(1-\lambda_{m}^{\alpha}\right) d^{\alpha}\right)}{\left(\varepsilon_{m}^{\alpha}+d^{\alpha}\right)\left(t_{2 m}^{\alpha}+\delta_{m}^{\alpha}+d^{\alpha}\right)+d^{\alpha} \gamma_{m}^{\alpha}}, \\
R_{0 x} & =\frac{\beta_{x}^{\alpha}\left(\varepsilon_{x}^{\alpha}+\left(1-\lambda_{x}^{\alpha}\right) d^{\alpha}\right)}{\left(\varepsilon_{x}^{\alpha}+d^{\alpha}\right)\left(t_{2 x}^{\alpha}+\delta_{x}^{\alpha}+d^{\alpha}\right)+d^{\alpha} \gamma_{x}^{\alpha}} .
\end{aligned}
$$

\section{Equilibrium Points and Their Asymptotic Stability}

To discuss the local asymptotic stability for evaluating the equilibrium points, let us consider the following [38]:

$$
\begin{aligned}
D_{t}^{\alpha} S & =D_{t}^{\alpha} L_{s}=D_{t}^{\alpha} L_{m}=D_{t}^{\alpha} L_{x}=D_{t}^{\alpha} I_{s}=D_{t}^{\alpha} I_{m} \\
& =D_{t}^{\alpha} I_{x}=D_{t}^{\alpha} R=0 .
\end{aligned}
$$

Then, from (A.1)

$$
g_{i}\left(\bar{S}, \overline{L_{s}}, \overline{L_{m}}, \overline{L_{x}}, \overline{I_{s}}, \overline{I_{m}}, \overline{I_{x}}, \bar{R}\right)=0, \quad i=1,2,3, \ldots, 8,
$$

where $\left(\bar{S}, \overline{L_{s}}, \overline{L_{m}}, \overline{L_{x}}, \overline{I_{s}}, \overline{I_{m}}, \overline{I_{x}}, \bar{R}\right)$ denotes any equilibrium point.

3.1. Stability of the Disease-Free Equilibrium. If $I_{s}(t)=I_{m}(t)=$ $I_{x}(t)=0$ then $L_{s}(t)=L_{m}(t)=L_{x}(t)=0, R(t)=0$, and 
$S(t)=b^{\alpha} / d^{\alpha}$. Then the disease-free equilibrium (DFE) is $E_{0}=$ $\left\{\left(b^{\alpha} / d^{\alpha}, 0,0,0,0,0,0,0\right)\right\}$.

Let us consider the coordinate transformation: $s(t)=$ $S(t)-\bar{S}, l_{s}(t)=L_{s}(t)-\overline{L_{s}}, l_{m}(t)=L_{m}(t)-\overline{L_{m}}, l_{x}(t)=$
$L_{x}(t)-\overline{L_{x}}, i_{s}(t)=I_{s}(t)-\overline{I_{s}}, i_{m}(t)=I_{m}(t)-\overline{I_{m}}, i_{x}(t)=$ $I_{x}(t)-\bar{I}_{x}, r(t)=R(t)-\bar{R}$. The corresponding characteristic equation for DFE is given as follows:

$$
\left(J\left(E_{0}\right)-\lambda I\right)=\left(\begin{array}{cccccccc}
\lambda-a & 0 & 0 & 0 & b & c & d_{1} & 0 \\
0 & \lambda-e & 0 & 0 & f & 0 & 0 & 0 \\
0 & g & \lambda-h & 0 & p & q & 0 & 0 \\
0 & 0 & 0 & \lambda-r & 0 & s & t & 0 \\
0 & u & 0 & 0 & \lambda-v & 0 & 0 & 0 \\
0 & 0 & w & 0 & 0 & \lambda-x+t_{2 m}^{\alpha} e^{(-\lambda \tau)} & 0 & 0 \\
0 & 0 & 0 & y & 0 & 0 & \lambda-z+t_{2 x}^{\alpha} e^{(-\lambda \tau)} & 0 \\
0 & m & 0 & 0 & n & j & t_{2 x}^{\alpha} e^{(-\lambda \tau)} & \lambda-a
\end{array}\right),
$$

where $a=-d^{\alpha}, b=-\beta_{s}^{\alpha}, c=-\beta_{m}^{\alpha}, d_{1}=-\beta_{x}^{\alpha}, e=-\left(d^{\alpha}+\right.$ $\left.\varepsilon_{s}^{\alpha}+t_{1 s}^{\alpha}\right), f=\gamma_{s}^{\alpha}+\lambda_{s}^{\alpha} \beta_{s}^{\alpha}, g=\left(1-p_{1}^{\alpha}\right) t_{1 s}^{\alpha}, h=-\left(d^{\alpha}+\varepsilon_{m}^{\alpha}\right), p=$ $\left(1-p_{2}^{\alpha}\right) t_{2 s}^{\alpha}, q=\gamma_{m}^{\alpha}+\lambda_{m}^{\alpha} \beta_{m}^{\alpha}, r=-\left(d^{\alpha}+\varepsilon_{x}^{\alpha}\right), s=\left(1-p_{3}^{\alpha}\right) t_{2 m}^{\alpha}, t=$ $\gamma_{x}^{\alpha}+\lambda_{x}^{\alpha} \beta_{x}^{\alpha}, u=\varepsilon_{s}^{\alpha}, v=-\left(d^{\alpha}+\delta_{s}^{\alpha}+t_{2 s}^{\alpha}+\gamma_{s}^{\alpha}\right), w=\varepsilon_{m}^{\alpha}, x=$ $-\left(d^{\alpha}+\delta_{m}^{\alpha}+\gamma_{m}^{\alpha}\right), y=\varepsilon_{x}^{\alpha}, z=-\left(d^{\alpha}+\delta_{x}^{\alpha}+\gamma_{x}^{\alpha}\right), m=p_{1}^{\alpha} t_{1 s}^{\alpha}, n=$ $p_{2}^{\alpha} t_{2 s}^{\alpha}, j=p_{3}^{\alpha} t_{2 m}^{\alpha}$. is [38]

The characteristic equation associated with above matrix

$$
\begin{aligned}
& \Delta(\lambda)=\left|J\left(E_{0}\right)-\lambda I\right|=0, \\
& (a-\lambda)^{2}\left(\lambda^{2}-\left(r+z+t_{2 x}^{\alpha} e^{(-\lambda \tau)}\right) \lambda-y t\right. \\
& \left.+\left(z-t_{2 x}^{\alpha} e^{(-\lambda \tau)}\right) r\right)\left(-\lambda^{2}+\left(h+x-t_{2 m}^{\alpha} e^{(-\lambda \tau)}\right) \lambda\right. \\
& \left.-\left(x+t_{2 m}^{\alpha} e^{(-\lambda \tau)}\right) h+w q\right)\left(-\lambda^{2}+(e+v) \lambda+u f\right. \\
& -v e)=0 .
\end{aligned}
$$

Lemma 1. If $R_{0}<1$, then the disease-free equilibrium $E_{0}$ is locally asymptotically stable for $\tau=0$.

Proof. When $\tau=0$, the associated transcendental characteristic equation $\Delta(\lambda)$ of system (1) at $E_{0}$ becomes $\Delta(\lambda)=$ $P(\lambda)=0$, and then the eigenvalues of the Jacobian matrix are

$$
\begin{aligned}
& \lambda_{1,2}=-d, \\
& \lambda_{3,4} \\
& =\frac{r+\left(z-t_{2 x}^{\alpha}\right) \pm \sqrt{\left(r^{2}-2\left(z-t_{2 x}^{\alpha}\right) r+\left(z-t_{2 x}^{\alpha}\right)^{2}+4 y t\right)}}{2},
\end{aligned}
$$

$$
\begin{aligned}
& \lambda_{5,6} \\
& =\frac{x-t_{2 m}^{\alpha}+h \pm \sqrt{\left(x-t_{2 m}^{\alpha}\right)^{2}-2\left(x-t_{2 m}^{\alpha}\right) h+h^{2}+4 w q}}{2}, \\
& \lambda_{7,8}=\frac{v+e \pm \sqrt{\left(v^{2}-2 v e+e^{2}+4 u f\right)}}{2},
\end{aligned}
$$

and by using Routh Hurwitz Theorem [28], these roots are negative or have negative real parts and all eigenvalues satisfy Matignon's conditions [39], given by $\left(\left|\arg \lambda_{i}\right|>\alpha \pi / 2\right)$ so the disease-free equilibrium $E_{0}$ is locally asymptotically stable.

Lemma 2. Let $R_{0}<1$, and then the disease-free equilibrium $E_{0}$ is locally asymptotically stable for $\tau>0$.

Proof. Let us consider $\tau>0$, and we noted that second and third factor of the characteristic equation (12), which are $\left(\lambda^{2}-\right.$ $\left.\left(r+z+t_{2 x}^{\alpha} e^{(-\lambda \tau)}\right) \lambda-y t+\left(z-t_{2 x}^{\alpha} e^{(-\lambda \tau)}\right) r\right)$ and $\left(-\lambda^{2}+(h+x-\right.$ $\left.\left.t_{2 m}^{\alpha} e^{(-\lambda \tau)}\right) \lambda-\left(x+t_{2 m}^{\alpha} e^{(-\lambda \tau)}\right) h+w q\right)$, have no pure imaginary roots for any value of the delay $\tau$, if $R_{0}<1$. Hence all the roots of the characteristic equation have negative real parts and we get that DFE is locally asymptotically stable regardless of the value of the delay and all eigenvalues satisfy Matignon's conditions [39], given by $\left(\left|\arg \lambda_{i}\right|>\alpha \pi / 2\right)$ so the disease-free equilibrium $E_{0}$ is locally asymptotically stable.

3.2. Stability of the Endemic Equilibrium. System (1) has an endemic equilibrium if at least one of the infected variables is not zero. The expression "analytic" is complexity and not useful for our purposes. Consider the values of parameters from Table 3. Then the basic reproduction number is $R_{0}>1$. The endemic equilibrium $S=338.2, L_{s}=0, L_{m}=0, L_{x}=$ 2233.8, $I_{s}=0, I_{m}=0, I_{x}=4820.6, R=62.0$. The matrices 
$A_{1}$ and $A_{2}$ associated with the linearized system at the endemic equilibrium are computed as

$$
A_{1}=\left(\begin{array}{ccccccccc}
-9.0226 & 0.4107 & 0.4107 & 0.4107 & -0.2244 & -0.2244 & -0.2244 & 0.4107 \\
0 & -2.2127 & 0 & 0 & 0.6321 & 0 & 0 & 0 \\
0 & 0.8800 & -1.3327 & 0 & 0.800 & 0.6321 & 0 & 0 \\
4.4475 & 0.1472 & 0.1472 & -1.4118 & -0.0791 & -0.0750 & 0.3432 & 1.0525 \\
0 & 0.5000 & 0 & 0 & -1.2729 & 0 & 0 & 0 \\
0 & 0.0146 & 0.5 & 0 & 0 & -0.4415 & 0 & 0 \\
4.1762 & -0.1241 & -0.3181 & 0.6023 & -0.3504 & -0.3504 & -0.5675 & 0.7813 \\
0.0188 & 0.0188 & 0.0188 & 0.0188 & -0.0103 & 0.0196 & 0.0237 & -2.6245
\end{array}\right),
$$

The transcendental characteristic equation $\Delta \lambda=\left(\lambda I-A_{1}-\right.$ $e^{-\tau \lambda} A_{2}$ ) is given by

$$
\begin{aligned}
\lambda^{8} & +\left(18.8862-0.0680 e^{-\tau \lambda}\right) \lambda^{7} \\
& +\left(1.2702 \times 10^{2}-1.2255 e^{-\tau \lambda}+0.0012 e^{-2 \tau \lambda}\right) \lambda^{6} \\
& +\left(4.2273 \times 10^{2}-7.5306 e^{-\tau \lambda}+0.0198 e^{-2 \tau \lambda}\right) \lambda^{5} \\
& +\left(7.7354 \times 10^{2}-22.1163 e^{-\tau \lambda}+0.10986 e^{-2 \tau \lambda}\right) \lambda^{4} \\
& +\left(7.9327 \times 10^{2}-34.3423 e^{-\tau \lambda}+0.2751 e^{-2 \tau \lambda}\right) \lambda^{3} \\
& +\left(4.3522 \times 10^{2}-28.4367 e^{-\tau \lambda}+0.3354 e^{-2 \tau \lambda}\right) \lambda^{2} \\
& +\left(1.1156 \times 10^{2}-11.6693 e^{-\tau \lambda}+0.18817 e^{-2 \tau \lambda}\right) \\
& \cdot \lambda\left(9.6913-1.8329 e^{-\tau \lambda}+0.0367 e^{-2 \tau \lambda}\right)=0
\end{aligned}
$$

when $\tau=0$, and we have the following characteristic equation:

$$
\begin{aligned}
\lambda^{8}+ & 18.8182 \lambda^{7}+125.7957 \lambda^{6}+415.2192 \lambda^{5} \\
& +751.5336 \lambda^{4}+759.2028 \lambda^{3}+407.1187 \lambda^{2} \\
& +100.0789 \lambda+7.8951=0 .
\end{aligned}
$$

The roots of $(16)$ are $-0.14208,-0.41505,-1.57465,-1.40036$, $-1.01044,-9.15198,-2.56181+0.05148 i$, and $-2.56181+$ $0.05148 i$, and these roots are negative or have negative real parts and all eigenvalues satisfy Matignon's conditions [39], given by $\left(\left|\arg \lambda_{i}\right|>\alpha \pi / 2\right) i=1,2, \ldots, 8$ so the endemic equilibrium is locally asymptotically stable.

Consider now the case $\tau>0$; we noted that the roots of the characteristic equation (15) have no pure imaginary roots for any value of the delay $\tau$, if $R_{0}>1$. Hence all the roots of the characteristic equation have negative real parts and all eigenvalues satisfy Matignon's conditions [39]. Therefore, the endemic equilibrium is locally asymptotically.

\section{NSFD for Fractional Delay Differential Equations}

In this section, we apply NSFD method with GLFDs to obtain the discretization of the delay fractional multistrain TB model (1), which will yield the following equations:

$$
\begin{aligned}
& \sum_{j=0}^{n+1} \omega_{j}^{\alpha} S^{n+1-j}=b^{\alpha}-d^{\alpha} S^{n+1}-\beta_{s}^{\alpha} \frac{S^{n+1} I_{s}^{n}}{N^{n}}-\beta_{m}^{\alpha} \frac{S^{n+1} I_{m}^{n}}{N^{n}} \\
& \quad-\beta_{x}^{\alpha} \frac{S^{n+1} I_{x}^{n}}{N^{n}},
\end{aligned}
$$


Complexity

7

$$
\begin{aligned}
& \sum_{j=0}^{n+1} \omega_{j}^{\alpha} L_{s}^{n+1-j}=\lambda_{s}^{\alpha} \beta_{s}^{\alpha} \frac{S^{n+1} I_{s}^{n}}{N^{n}}+\sigma_{s}^{\alpha} \lambda_{s}^{\alpha} \beta_{s}^{\alpha} \frac{R^{n+1} I_{s}^{n}}{N^{n}}+\gamma_{s}^{\alpha} I_{s}^{n} \\
& -\alpha_{s s}^{\alpha} \beta_{s} \frac{L_{s}^{n+1} I_{s}^{n}}{N^{n}}-\alpha_{s x}^{\alpha} \beta_{x}^{\alpha} \frac{L_{s}^{n+1} I_{x}^{n}}{N^{n}}-\left(d^{\alpha}+\varepsilon_{s}^{\alpha}+t_{1 s}^{\alpha}\right) \\
& \cdot L_{s}^{n+1}-\alpha_{s m}^{\alpha} \beta_{m}^{\alpha} \frac{L_{s}^{n+1} I_{m}^{n}}{N^{n}} \\
& \sum_{j=0}^{n+1} \omega_{j}^{\alpha} L_{m}^{n+1-j}=\lambda_{m}^{\alpha} \beta_{m}^{\alpha} \frac{S^{n+1} I_{m}^{n}}{N^{n}}+\sigma_{m}^{\alpha} \lambda_{m}^{\alpha} \beta_{m}^{\alpha} \frac{R^{n+1} I_{m}^{n}}{N^{n}} \\
& +\lambda_{m}^{\alpha} \alpha_{s m}^{\alpha} \beta_{m}^{\alpha} \frac{L_{s}^{n+1} I_{m}^{n}}{N^{n}}+\gamma_{m}^{\alpha} I_{m}^{n}+t_{1 s}^{\alpha} L_{s}^{n+1}-P_{1}^{\alpha} t_{1 s}^{\alpha} L_{s}^{n+1} \\
& +t_{2 s}^{\alpha} I_{s}^{n}-P_{2}^{\alpha} t_{2 s}^{\alpha} I_{s}^{n}-\alpha_{m m}^{\alpha} \beta_{m}^{\alpha} \frac{L_{m}^{n+1} I_{m}^{n}}{N^{n}}-\alpha_{m x}^{\alpha} \beta_{x}^{\alpha} \frac{L_{m}^{n+1} I_{x}^{n}}{N^{n}} \\
& -\left(d^{\alpha}+\varepsilon_{m}^{\alpha}\right) L_{m}^{n+1}, \\
& \sum_{j=0}^{n+1} \omega_{j}^{\alpha} L_{x}^{n+1-j}=\lambda_{x}^{\alpha} \beta_{x}^{\alpha} \frac{S^{n+1} I_{x}^{n}}{N^{n}}+\sigma_{x}^{\alpha} \lambda_{x}^{\alpha} \beta_{x}^{\alpha} \frac{R^{n+1} I_{x}^{n}}{N^{n}} \\
& +\lambda_{x}^{\alpha} \alpha_{s x}^{\alpha} \beta_{x}^{\alpha} \frac{L_{s}^{n+1} I_{x}^{n}}{N^{n}}+\gamma_{x}^{\alpha} I_{x}^{n}+\lambda_{x}^{\alpha} \alpha_{m x}^{\alpha} \beta_{x}^{\alpha} \frac{L_{m}^{n+1} I_{x}^{n}}{N^{n}} \\
& +t_{2 m}^{\alpha} I_{m}^{n}-P_{3}^{\alpha} t_{2 m}^{\alpha} I_{m}^{n}-\alpha_{x x}^{\alpha} \beta_{x}^{\alpha} \frac{L_{x}^{n+1} I_{x}^{n}}{N^{n}}-\left(d^{\alpha}+\varepsilon_{x}^{\alpha}\right) \\
& \cdot L_{x}^{n+1} \\
& \sum_{j=0}^{n+1} \omega_{j}^{\alpha} I_{s}^{n+1-j}=\alpha_{s s}^{\alpha} \beta_{s}^{\alpha} \frac{L_{s}^{n+1} I_{s}^{n}}{N^{n}}+\left(1-\lambda_{s}^{\alpha}\right) \\
& \cdot \beta_{s}^{\alpha}\left(\frac{S^{n+1} I_{s}^{n}}{N^{n}}+\sigma_{s}^{\alpha} \frac{R^{n+1} I_{s}^{n}}{N^{n}}\right)+\varepsilon_{s}^{\alpha} L_{s}^{n+1}-\left(d^{\alpha}+\delta_{s}^{\alpha}\right) \\
& \cdot I_{s}^{n+1}-\left(\gamma_{s}^{\alpha}+t_{2 s}^{\alpha}\right) I_{s}^{n}, \\
& \sum_{j=0}^{n+1} \omega_{j}^{\alpha} I_{m}^{n+1-j}=\alpha_{m m}^{\alpha} \beta_{m}^{\alpha} \frac{L_{m}^{n+1} I_{m}^{n}}{N^{n}}+\left(1-\lambda_{m}^{\alpha}\right) \\
& \cdot \beta_{m}^{\alpha}\left(\frac{S^{n+1} I_{m}^{n}}{N^{n}}+\sigma_{m}^{\alpha} \frac{R^{n+1} I_{m}^{n}}{N^{n}}+\alpha_{s m}^{\alpha} \frac{L_{s}^{n+1} I_{m}^{n}}{N^{n}}\right) \\
& +\varepsilon_{m}^{\alpha} L_{m}^{n+1}-\left(d^{\alpha}+\delta_{m}^{\alpha}\right) I_{m}^{n+1}-\gamma_{m}^{\alpha} I_{m}-t_{2 m}^{\alpha} I_{m}^{n-\kappa}, \\
& \sum_{j=0}^{n+1} \omega_{j}^{\alpha} I_{x}^{n+1-j}=\alpha_{x x}^{\alpha} \beta_{x}^{\alpha} \frac{L_{x}^{n+1} I_{x}^{n}}{N^{n}}+\left(1-\lambda_{x}^{\alpha}\right)
\end{aligned}
$$

$$
\begin{aligned}
S^{n+1} & =\frac{b^{\alpha}-\sum_{j=1}^{n+1} \omega_{j}^{\alpha} S^{n+1-j}}{\left(\varphi_{1}(h)\right)^{-\alpha}+d^{\alpha}+\left(\beta_{s}^{\alpha} I_{s}^{n}+\beta_{m}^{\alpha} I_{m}^{n}+\beta_{x}^{\alpha} I_{x}^{n}\right) / N^{n}}, \\
L_{s}^{n+1} & =\frac{\left(\beta_{s}^{\alpha} I_{s}^{n} / N^{n}\right) \lambda_{s}^{\alpha}\left(S^{n+1}+\sigma_{s}^{\alpha} R^{n+1}\right)+\gamma_{s}^{\alpha} I_{s}^{n}-\sum_{j=1}^{n+1} \omega_{j}^{\alpha} L_{s}^{n+1-j}}{\left(\varphi_{2}(h)\right)^{-\alpha}+\left(d^{\alpha}+t_{1 s}^{\alpha}+\varepsilon_{s}^{\alpha}\right)+\left(1 / N^{n}\right)\left(\alpha_{s s}^{\alpha} \beta_{s}^{\alpha} I_{s}^{n}+\alpha_{s m}^{\alpha} \beta_{m}^{\alpha} I_{m}^{n}+\alpha_{s x}^{\alpha} \beta_{x}^{\alpha} I_{x}^{n}\right)},
\end{aligned}
$$

Then we obtain

$$
\begin{aligned}
& \cdot \beta_{m}\left(\frac{S^{n+1} I_{x}^{n}}{N^{n}}+\sigma_{x} \frac{R^{n+1} I_{x}^{n}}{N^{n}}+\alpha_{m x} \frac{L_{x}^{n+1} I_{m}^{n}}{N^{n}}\right) \\
& +\varepsilon_{x}^{\alpha} L_{x}^{n+1}-\left(d^{\alpha}+\delta_{x}^{\alpha}\right) I_{x}^{n+1}-\gamma_{x}^{\alpha} I_{x}-t_{2 x}^{\alpha} I_{x}^{n-\kappa},
\end{aligned}
$$

$$
\begin{aligned}
& \sum_{j=0}^{n+1} \omega_{j}^{\alpha} R^{n+1-j}=P_{1}^{\alpha} t_{1 s}^{\alpha} L_{s}^{n+1}+P_{2}^{\alpha} t_{2 s}^{\alpha} I_{s}^{n}+P_{3}^{\alpha} t_{2 m}^{\alpha} I_{m}^{n} \\
& \quad+t_{2 x}^{\alpha} I_{x}^{n-\kappa}-d^{\alpha} R^{n+1}-\sigma_{s}^{\alpha} \beta_{s}^{\alpha} \frac{R^{n+1} I_{s}^{n}}{N^{n}}-\sigma_{m}^{\alpha} \beta_{m}^{\alpha} \frac{R^{n+1} I_{m}^{n}}{N^{n}} \\
& \quad-\sigma_{x}^{\alpha} \beta_{x}^{\alpha} \frac{R^{n+1} I_{x}^{n}}{N^{n}}
\end{aligned}
$$

where

$$
N^{n}=S^{n}+L_{s}^{n}+L_{m}^{n}+L_{x}^{n}+I_{s}^{n}+I_{m}^{n}+I_{x}^{n}+R^{n}
$$

and $\omega_{0}^{\alpha}=\left(\varphi_{i}(h)\right)^{-\alpha}, i=1,2, \ldots, 8, n=-\kappa,-\kappa+1, \ldots, 0,1$, where the nonlocal approximations are used for the nonlinear terms and the following functions of denominator:

$$
\begin{aligned}
& \varphi_{1}(h)=\frac{e^{d^{\alpha} h}-1}{d^{\alpha}}, \\
& \varphi_{2}(h)=\frac{e^{\left(d^{\alpha}+\varepsilon_{s}^{\alpha}+t_{1 s}^{\alpha}\right) h}-1}{\left(d^{\alpha}+\varepsilon_{s}^{\alpha}+t_{1 s}^{\alpha}\right)}, \\
& \varphi_{3}(h)=\frac{e^{\left(d^{\alpha}+\varepsilon_{m}^{\alpha}\right) h}-1}{\left(d^{\alpha}+\varepsilon_{m}^{\alpha}\right)} \\
& \varphi_{4}(h)=\frac{e^{\left(d^{\alpha}+\varepsilon_{x}^{\alpha}\right) h}-1}{\left(d^{\alpha}+\varepsilon_{x}^{\alpha}\right)} \\
& \varphi_{5}(h)=\frac{1-e^{-\left(d^{\alpha}+\delta_{s}^{\alpha}\right) h}}{\left(\gamma_{s}^{\alpha}+t_{2 s}^{\alpha}\right)}, \\
& \varphi_{6}(h)=\frac{1-e^{-\left(d^{\alpha}+\delta_{m}^{\alpha}\right) h}}{\left(\gamma_{m}^{\alpha}+t_{2 m}^{\alpha}\right)}, \\
& \varphi_{7}(h)=\frac{1-e^{-\left(d^{\alpha}+\delta_{x}^{\alpha}\right) h}}{\left(\gamma_{x}^{\alpha}+t_{2 x}^{\alpha}\right)}, \\
& \varphi_{8}(h)=\frac{e^{d^{\alpha} h}-1}{d^{\alpha}} .
\end{aligned}
$$




$$
\begin{aligned}
& L_{m}^{n+1}=\frac{\left(\beta_{m}^{\alpha} \lambda_{m}^{\alpha} I_{m}^{n} / N^{n}\right)\left(S^{n+1}+\sigma_{m}^{\alpha} R^{n+1}+\alpha_{s m}^{\alpha} L_{s}^{n+1}\right)+\gamma_{m}^{\alpha} I_{m}^{n}+t_{1 s}^{\alpha} L_{s}^{n+1}\left(1-P_{1}^{\alpha}\right)}{\left(\varphi_{3}(h)\right)^{-\alpha}+\left(d^{\alpha}+\varepsilon_{m}^{\alpha}\right)+\left(1 / N^{n}\right)\left(\alpha_{m m}^{\alpha} \beta_{m}^{\alpha} I_{m}^{n}+\alpha_{m x}^{\alpha} \beta_{x}^{\alpha} I_{x}^{n}\right)} \\
& +\frac{t_{2 s}^{\alpha} I_{s}^{n}\left(1-P_{2}^{\alpha}\right)-\sum_{j=1}^{n+1} \omega_{j}^{\alpha} L_{m}^{n+1-j}}{\left(\varphi_{3}(h)\right)^{-\alpha}+\left(d^{\alpha}+\varepsilon_{m}^{\alpha}\right)+\left(1 / N^{n}\right)\left(\alpha_{m m}^{\alpha} \beta_{m}^{\alpha} I_{m}^{n}+\alpha_{m x}^{\alpha} \beta_{x}^{\alpha} I_{x}^{n}\right)} \\
& L_{x}^{n+1}=\frac{\left(\beta_{x}^{\alpha} \lambda_{x}^{\alpha} I_{x}^{n} / N^{n}\right)\left(S^{n+1}+\sigma_{x}^{\alpha} R^{n+1}+\alpha_{s x}^{\alpha} L_{s}^{n+1}+\alpha_{m x}^{\alpha} L_{m}^{n+1}\right)+t_{2 m}^{\alpha} I_{m}^{n}\left(1-P_{3}^{\alpha}\right)}{\left(\varphi_{4}(h)\right)^{-\alpha}+\left(d^{\alpha}+\varepsilon_{x}^{\alpha}\right)+\left(1 / N^{n}\right)\left(\alpha_{x x}^{\alpha} \beta_{x}^{\alpha} I_{x}^{n}\right)} \\
& +\frac{\gamma_{x}^{\alpha} I_{x}^{n}-\sum_{j=1}^{n+1} \omega_{j}^{\alpha} L_{x}^{n+1-j}}{\left(\varphi_{4}(h)\right)^{-\alpha}+\left(d^{\alpha}+\varepsilon_{x}^{\alpha}\right)+\left(1 / N^{n}\right)\left(\alpha_{x x}^{\alpha} \beta_{x}^{\alpha} I_{x}^{n}\right)} \\
& I_{s}^{n+1}=\frac{\varphi_{5}(h) \beta_{s}^{\alpha}\left(I_{s}^{n} / N^{n}\right)\left(\alpha_{s s}^{\alpha} L_{s}^{n+1}+\left(1-\lambda_{s}^{\alpha}\right)\left(S^{n+1}+\sigma_{s}^{\alpha} R^{n+1}\right)\right)}{\left(\varphi_{5}(h)\right)^{-\alpha}+\left(d^{\alpha}+\delta_{s}^{\alpha}\right)}-\frac{\left(\gamma_{s}^{\alpha}+\left(t_{2 s}^{\alpha}\right)\right) I_{s}^{n}+\varepsilon_{s}^{\alpha} L_{s}^{n+1}-\sum_{j=1}^{n+1} \omega_{j}^{\alpha} I_{s}^{n+1-j}}{\left(\varphi_{5}(h)\right)^{-\alpha}+\left(d^{\alpha}+\delta_{s}^{\alpha}\right)} \\
& I_{m}^{n+1}=\frac{\beta_{m}^{\alpha}\left(I_{m}^{n} / N^{n}\right)\left(\alpha_{m m}^{\alpha} L_{m}^{n+1}+\left(1-\lambda_{m}^{\alpha}\right)\left(S^{n+1}+\sigma_{m}^{\alpha} R^{n+1}+\alpha_{s m}^{\alpha} L_{s}^{n+1}\right)\right)}{\left(\varphi_{6}(h)\right)^{-\alpha}+\left(d^{\alpha}+\delta_{m}^{\alpha}\right)}-\frac{\gamma_{m} I_{m}^{n}-t_{2 m}^{\alpha} I_{m}^{n-\kappa}+\varepsilon_{m}^{\alpha} L_{m}^{n+1}-\sum_{j=1}^{n+1} \omega_{j}^{\alpha} I_{m}^{n+1-j}}{\left(\varphi_{6}(h)\right)^{-\alpha}+\left(d^{\alpha}+\delta_{m}^{\alpha}\right)} \\
& I_{x}^{n+1}=\frac{\beta_{x}^{\alpha}\left(I_{x}^{n} / N^{n}\right)\left(\alpha_{x x}^{\alpha} L_{x}^{n+1}+\left(1-\lambda_{x}^{\alpha}\right)\left(S^{n+1}+\sigma_{x}^{\alpha} R^{n+1}+\alpha_{s x}^{\alpha} L_{s}^{n+1}+\alpha_{m x}^{\alpha} L_{m}^{n+1}\right)\right)}{\left(\varphi_{7}(h)\right)^{-\alpha}+\left(d^{\alpha}+\delta_{x}^{\alpha}\right)} \\
& -\frac{\gamma_{x}^{\alpha} I_{x}^{n}-t_{2 x}^{\alpha} I_{x}^{n-\kappa}+\varepsilon_{x}^{\alpha} L_{x}^{n+1}-\sum_{j=1}^{n+1} \omega_{j}^{\alpha} I_{x}^{n+1-j}}{\left(\varphi_{7}(h)\right)^{-\alpha}+\left(d^{\alpha}+\delta_{x}^{\alpha}\right)} \\
& R^{n+1}=\frac{t_{1 s}^{\alpha} P_{1}^{\alpha} L_{s}^{n+1}+P_{2}^{\alpha} t_{2 s}^{\alpha} I_{s}^{n}+t_{2 m}^{\alpha} P_{3}^{\alpha} I_{m}^{n}+t_{2 x}^{\alpha} I_{x}^{n-\kappa}-\sum_{j=1}^{n+1} \omega_{j}^{\alpha} R^{n+1-j}}{\left(\varphi_{8}(h)\right)^{-\alpha}+d^{\alpha}+\left(1 / N^{n}\right)\left(\sigma_{s}^{\alpha} \beta_{s}^{\alpha} I_{s}^{n}+\sigma_{m}^{\alpha} \beta_{m}^{\alpha} I_{m}^{n}+\sigma_{x}^{\alpha} \beta_{x}^{\alpha} I_{x}^{n}\right)}
\end{aligned}
$$

\section{Numerical Results and Simulations}

In this section, we show the effectiveness of the numerical technique for delay fractional differential equations. Throughout this section, all simulations are performed with initial conditions $\left(S(0), L_{s}(0), L_{m}(0), L_{x}(0), I_{s}(0), I_{m}(0)\right.$, $\left.I_{x}(0), R(0)\right)=(5000,50,50,50,30,30,30,60)$, with the parameters in Table 3 . The approximate solutions of the proposed system are given in Figures 1-12 at different values of $\tau$ and $\alpha$. Figure 1 shows the behavior of the approximate solutions of $R(t)$ in two cases with and without delay using dde23 at $\alpha=1$ and $\tau=0.3$. In Figure 2, we use the same data in Figure 1 and use NSFDM; we noted that the number of individuals $R(t)$ increases in the case of nondelay, that is, the delays in diagnosis and commencement of treatment to the individuals of $I_{m}$ and $I_{x}$ causing a shortage in the number of individuals of $R(t)$. Figure 3 shows the relationship between $I_{m}(t)$ and $I_{m}(t-\tau)$ and chaotic attractors at $\tau=0.1$ and $\alpha=1$. Figure 4 , shows the relationship between $I_{x}(t)$ and $I_{x}(t-\tau)$ at $\tau=0.1$ in case of integer order. Figures 5 and 6 show the relationship between $I_{m}(t)$ and $I_{m}(t-\tau)$ and $I_{x}(t)$ with $I_{x}(t-\tau)$, respectively, in case of fraction order where $\alpha=0.95$.
Figures 7 and 8 show the approximate solutions for $I_{m}(t-\tau)$ and $I_{x}(t-\tau)$ at $\tau=2, \alpha=0.98$ by using NSFDM. Figures 9 and 10 show the approximate solutions of different $\tau$ in both fraction and integer cases; we noted that increasing the value of $\tau$ causes decreasing the values of $R(t)$. Figures 11 and 12 show the behavior of the approximate solutions with different value of $\alpha$, which are given to demonstrate how the fractional model is a generalization of the integer order model.

\section{Conclusion}

Fractional models have the potential to describe more complex dynamics than the integer models and can include easily the memory effect present in many real world phenomena. In this paper, multistrain TB model of fractional order derivatives with time delay memory is presented. A nonstandard numerical scheme is introduced to numerically study the approximate solution of proposed model problem. The obtained results show that the delays in diagnosis and commencement of treatment to the individuals of $I_{m}$ and $I_{x}$ cause a shortage in the number of individuals of $R(t)$. The approximate solution of proposed model changes when $\tau$ and 


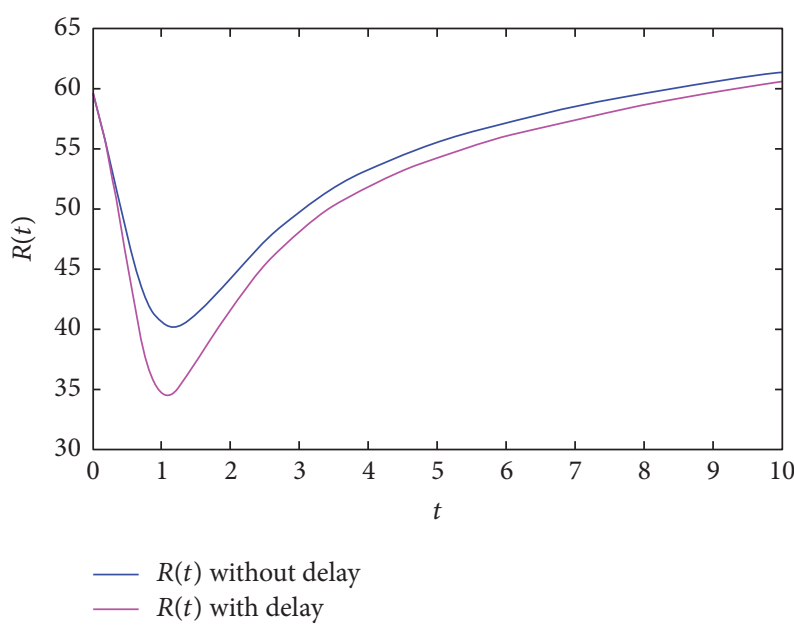

FIGURE 1: The approximate solution of $R(t)$ with $\tau=0.3$, using dde23.

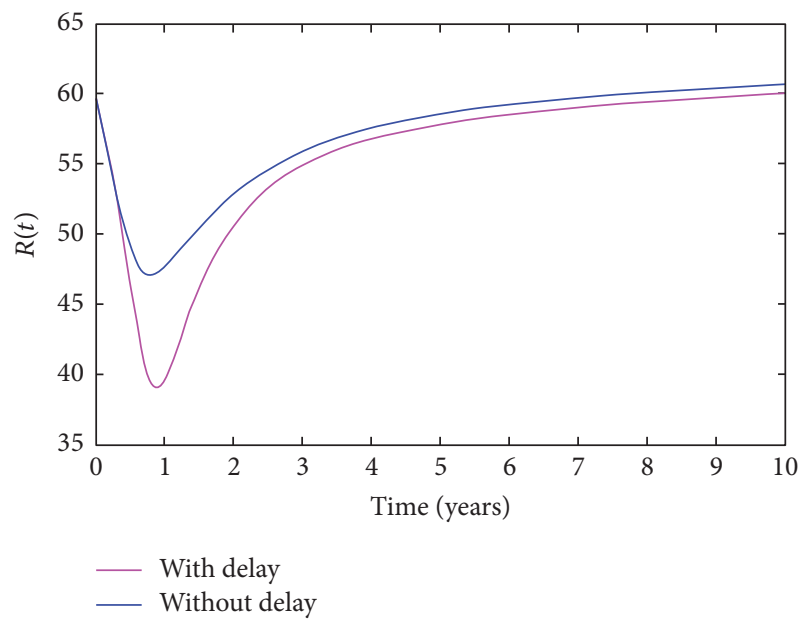

FIGURE 2: The approximate solution of $R(t)$ with $\tau=0.3$, using NSFDM, $\alpha=1$

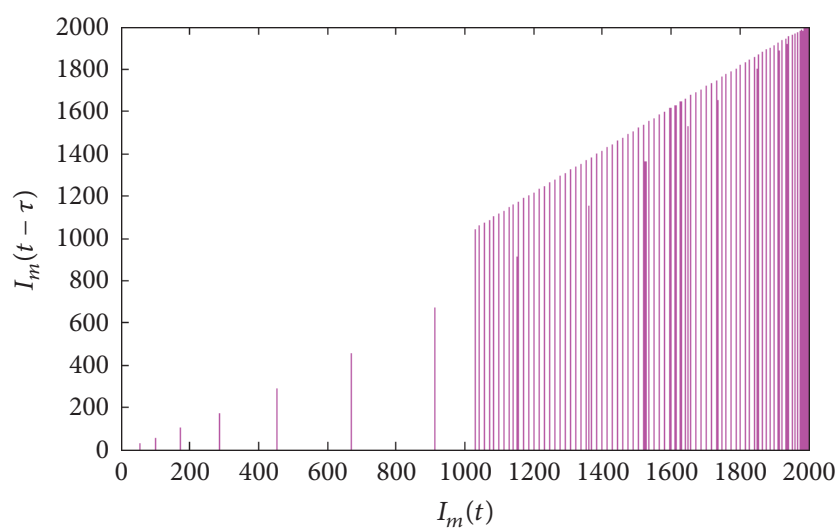

FIGURE 3: The relationship between $I_{m}(t), I_{m}(t-\tau)$ with $\tau=0.1, \alpha=$ 1 using NSFDM.
TABle 3: Parameter values of system (1).

\begin{tabular}{lc}
\hline Parameter & Value \\
\hline$b^{\alpha}$ & $3190^{\alpha}\left(\frac{1}{\text { year }}\right)^{\alpha}$ \\
$d^{\alpha}$ & $0.38^{\alpha}\left(\frac{1}{\text { year }}\right)^{\alpha}$ \\
$\beta_{s}^{\alpha}=\beta_{m}^{\alpha}=\beta_{x}^{\alpha}$ & $14^{\alpha}\left(\frac{1}{\text { year }}\right)^{\alpha}$ \\
$\lambda_{s}^{\alpha}=\lambda_{m}^{\alpha}=\lambda_{x}^{\alpha}$ & $0.5^{\alpha}\left(\frac{1}{\text { year }}\right)^{\alpha}$ \\
$\varepsilon_{s}^{\alpha}=\varepsilon_{m}^{\alpha}=\varepsilon_{x}^{\alpha}$ & $0.5^{\alpha}\left(\frac{1}{\text { year }}\right)^{\alpha}$ \\
$\alpha_{r 1, r 2}^{\alpha}$ & $0.05^{\alpha}\left(\frac{1}{\text { year }}\right)^{\alpha}$ \\
$\gamma_{s}^{\alpha}=\gamma_{m}^{\alpha}=\gamma_{x}^{\alpha}$ & $0.3^{\alpha}\left(\frac{1}{\text { year }}\right)^{\alpha}$ \\
$t_{1 s}^{\alpha}$ & $0.88^{\alpha}\left(\frac{1}{\text { year }}\right)^{\alpha}$ \\
$t_{2 r}^{\alpha}: r \in(s, m, x)$ & $t_{2 s}^{\alpha}=0.88^{\alpha} ;$ \\
& $t_{2 x}^{\alpha}=0.034^{\alpha}\left(\frac{1}{\text { year }}\right)^{\alpha}$ \\
$\sigma_{r}^{\alpha}$ & $0.25^{\alpha}\left(\frac{1}{\text { year }}\right)^{\alpha}$ \\
$P_{r}^{\alpha}$ & $0.88^{\alpha}\left(\frac{1}{\text { year }}\right)^{\alpha}$ \\
\hline$\delta_{r}^{\alpha}$ & $0.045^{\alpha}\left(\frac{1}{\text { year }}\right)^{\alpha}$ \\
\hline
\end{tabular}

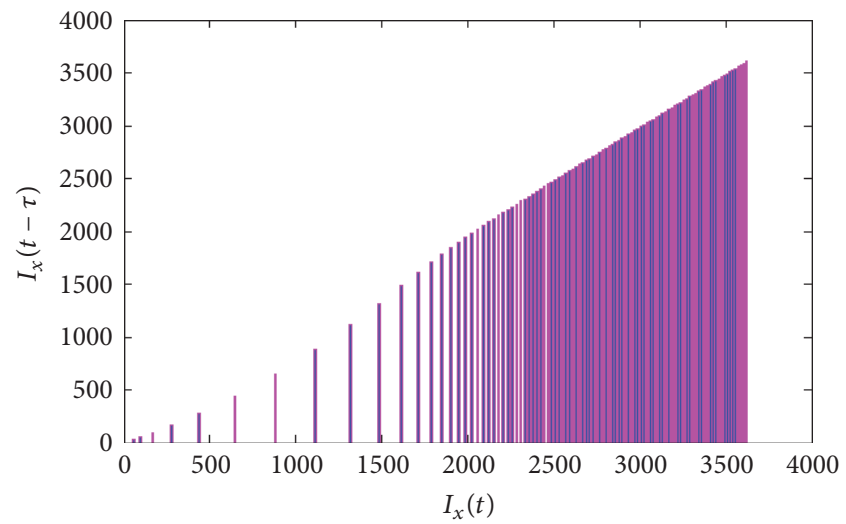

FIGURE 4: The relationship between $I_{x}(t), I_{x}(t-\tau)$ with $\tau=0.1, \alpha=$ 1 using NSFDM.

$\alpha$ take different values. Some figures are given to demonstrate how the fractional delay model is a generalization of the integer order model. It is concluded that NSFDM can be applied to solve such fractional delay differential equations simply and effectively. All results are obtained by using MATLAB programming. 


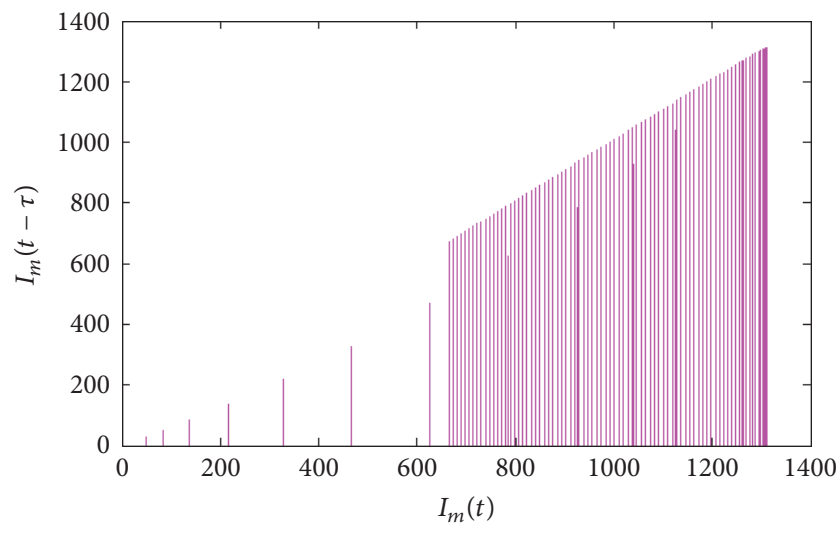

Figure 5: The relationship between $I_{m}(t), I_{m}(t-\tau)$ with $\tau=0.1, \alpha=$ 0.95 using NSFDM.

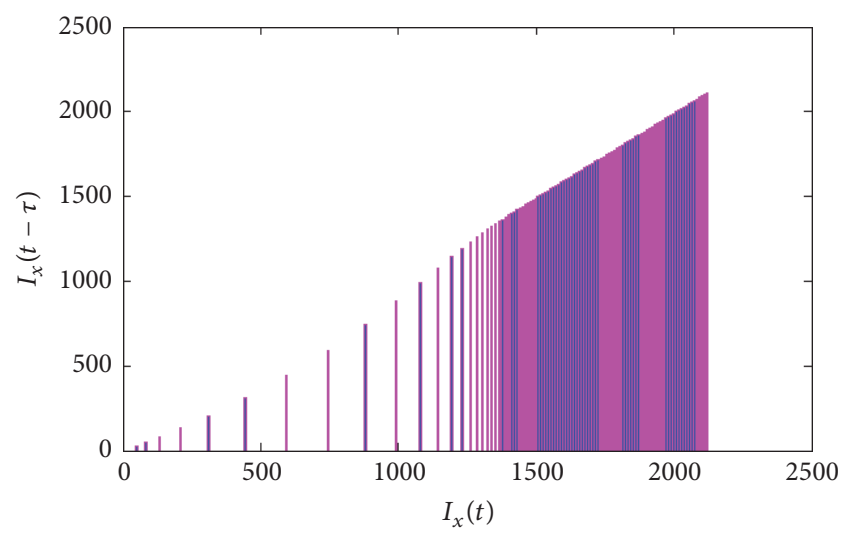

FIGURE 6: The relationship between $I_{x}(t), I_{x}(t-\tau)$ with $\tau=0.1, \alpha=$ 0.95 using NSFDM.

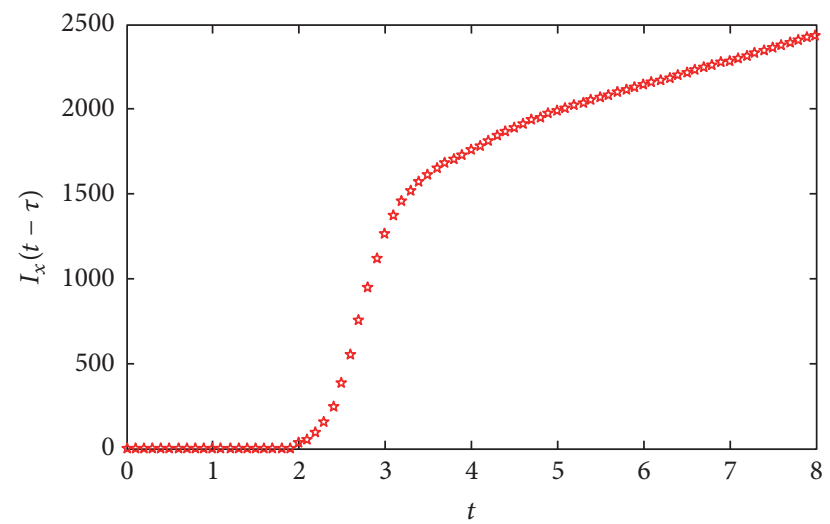

FIGURE 7: The approximate solutions $I_{x}(t-\tau)$ with $\tau=2, \alpha=0.98$ using NSFDM.

\section{Appendix}

\section{A. Preliminaries and Notations}

In this section, some basic definitions and properties in the theory of the fractional calculus are presented. Moreover,

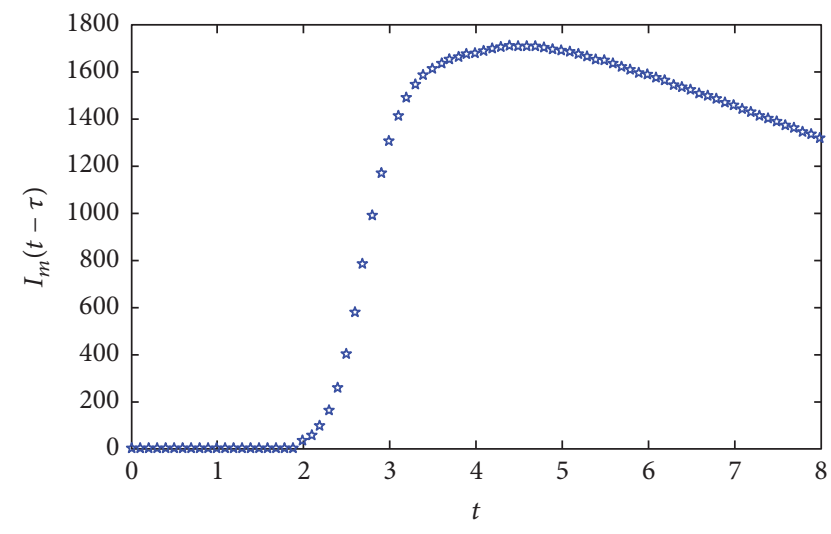

Figure 8: The approximate solutions $I_{m}(t-\tau)$ with $\tau=2, \alpha=0.98$ using NSFDM.
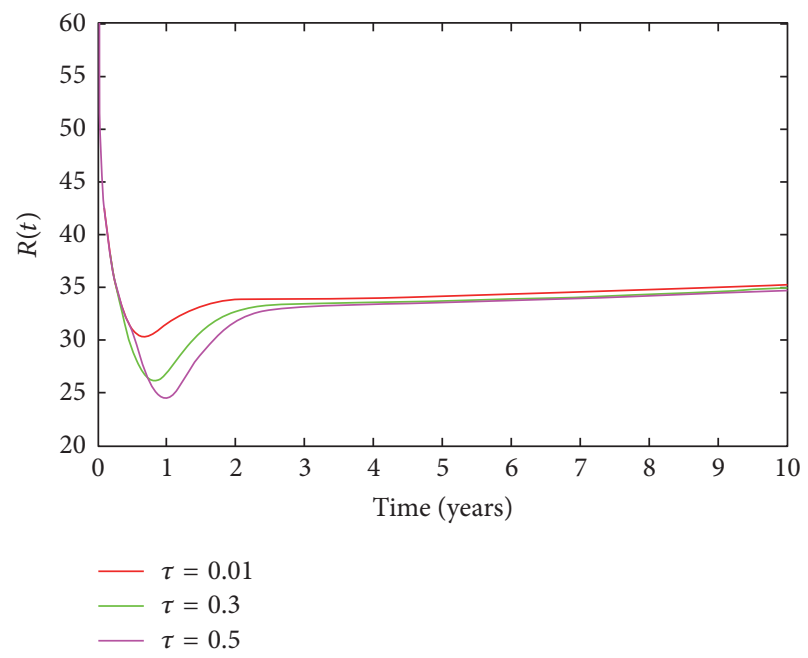

FIGURE 9: The approximate solutions with different $\tau$ and $\alpha=0.9$ using NSFDM.

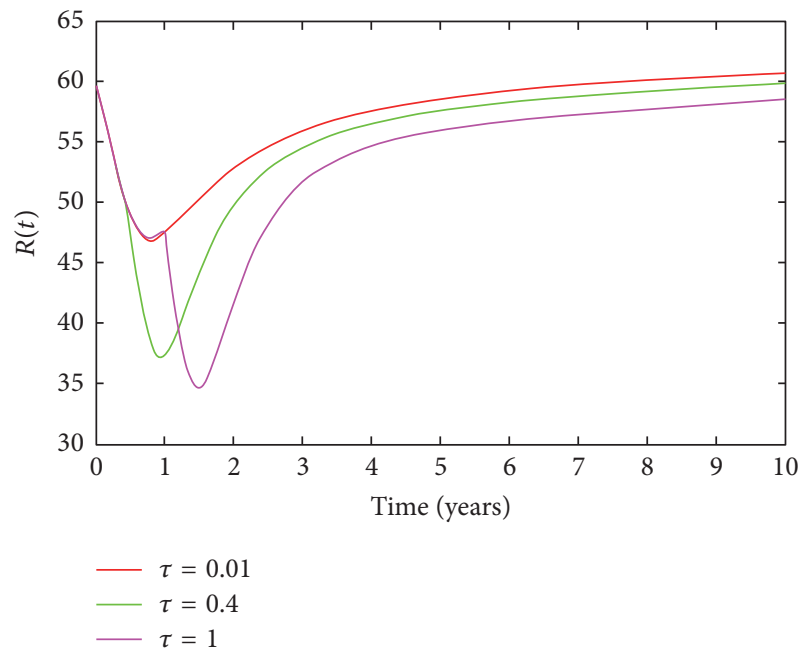

FIgURE 10: The approximate solution of $R(t)$ with different $\tau$, using NSFDM, $\alpha=1$. 

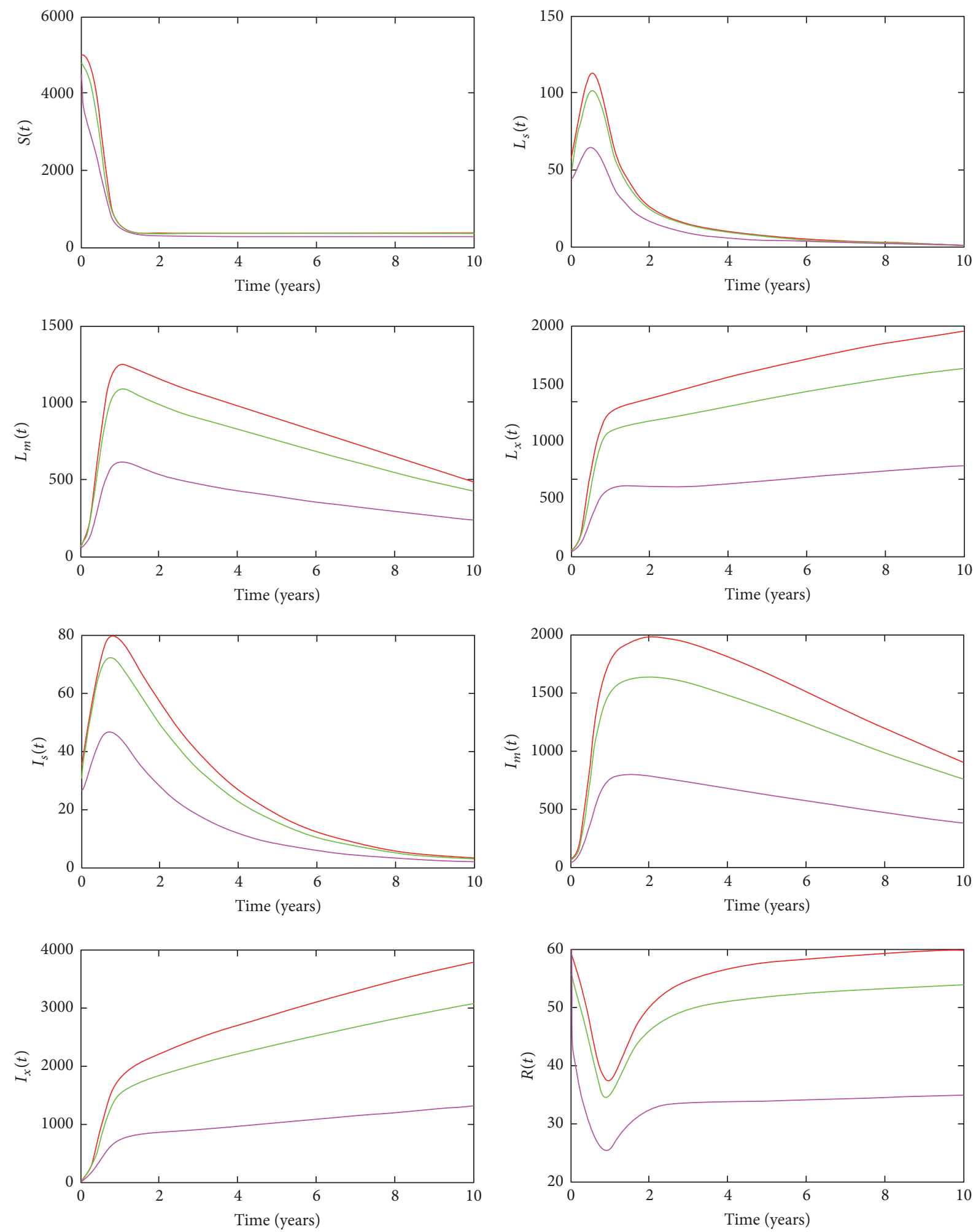

$\begin{aligned} \alpha & =1 \\ -\alpha & =0.98 \\ -\alpha & =0.90\end{aligned}$

$-\alpha=1$

$-\alpha=0.98$

$\alpha=0.90$

FIGURE 11: The approximate solutions with $\tau=0.4$ and different $\alpha$ using NSFDM. 

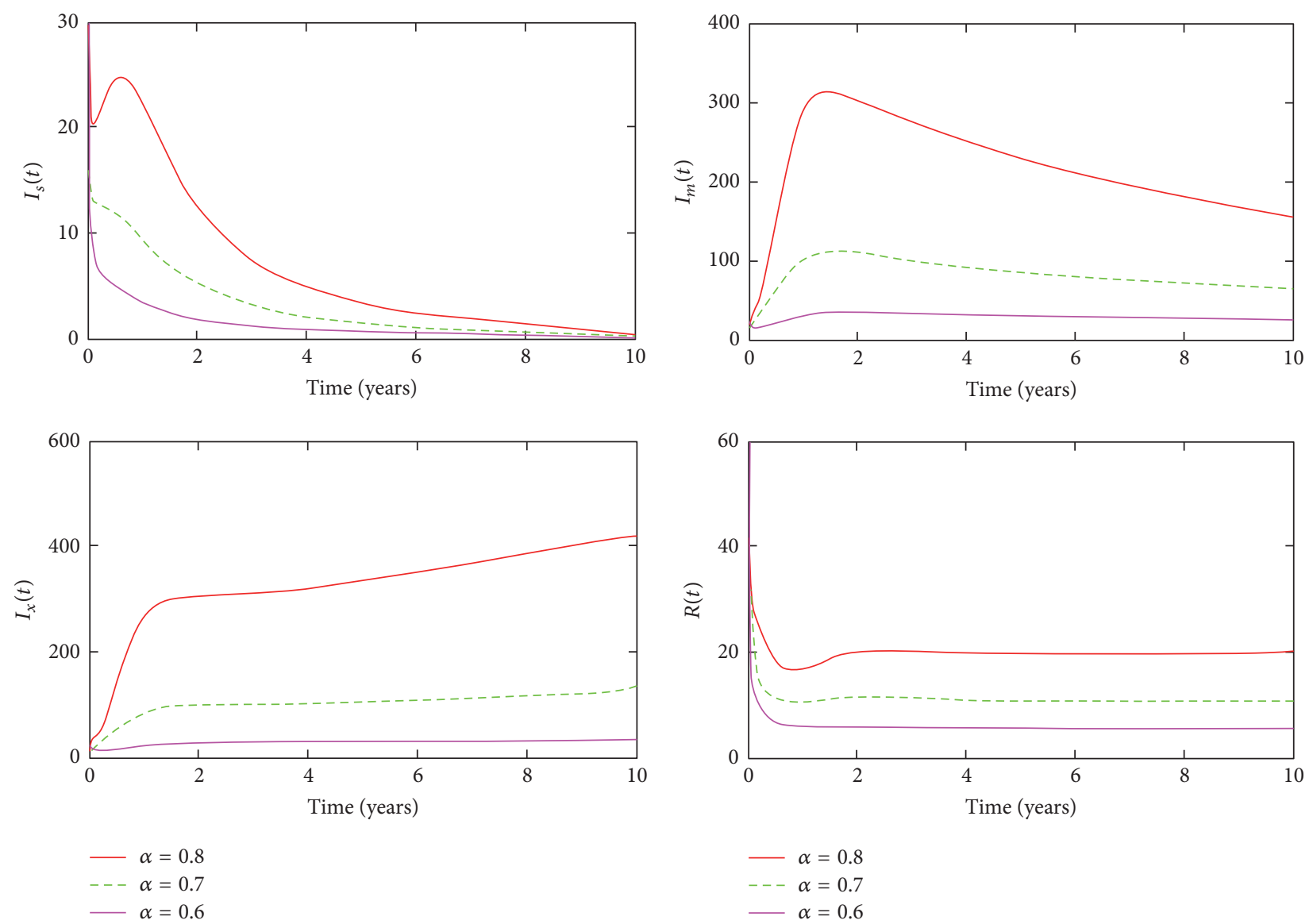

FIGURE 12: The approximate solutions with $\tau=0.4$ and different $\alpha$ using NSFDM.

we introduce the main aspects concerning nonstandard discretization methods.

A.1. Grünwald-Letinkov Fractional Derivatives (GLFDs). We will begin with the signal fractional differential equation (see $[17,40,41])$

$$
\begin{aligned}
D_{x}^{\alpha} z(x) & =g(x, z(x)), \quad T \geq x \geq 0, \\
z\left(x_{0}\right) & =0
\end{aligned}
$$

where $\alpha>0 . T$ is the final time and $D^{\alpha}$ denotes the fractional derivative, where $n-1<\alpha<n$, defined by

$$
D_{x}^{\alpha} z(x)=J^{n-\alpha} D_{x}^{\alpha} z(x)
$$

$\forall n \in \mathbb{N}$ and $J^{n}$ is the $n$ th-order Riemann-Liouville integral operator:

$$
J^{n} z(x)=\frac{1}{\Gamma(x)} \int_{0}^{x}(x-\tau)^{n-1} z(\tau) d \tau,
$$

with $\Gamma(\cdot)$ being the gamma function, and $x>0$. The Grünwald-Letinkov approximation of the fractional derivative is defined as follows [42]:

$$
D_{x}^{\alpha} z(x)=\lim _{h \rightarrow 0} h^{-\alpha} \sum_{r=0}^{m}(-1)^{r}\left(\begin{array}{l}
\alpha \\
r
\end{array}\right) z(x-r h),
$$

where $m=[x / h]$ denotes the integer part of $x / h$ and $h$ is the step-size. Equation (A.4) can be discretized as follows:

$$
\sum_{r=0}^{m} \omega_{r}^{\alpha} z\left(x_{n-r}\right)=g\left(x_{n}, z\left(x_{n}\right)\right) \quad n=1,2,3, \ldots,
$$

where $x_{n}=n h$ and $\omega_{r}^{\alpha}$ are the Grünwald-Letinkov coefficients defined as

$$
\omega_{r}^{\alpha}=\left(1-\frac{1+\alpha}{r}\right) \omega_{r-1}^{\alpha}, \quad \omega_{0}^{\alpha}=h^{-\alpha}, r=1,2,3, \ldots
$$

A.2. NSFD Discretization. It is known that the numerical scheme is called nonstandard method if at least one of the following conditions is satisfied [36]:

(1) the discretization of derivatives is not traditional and uses a nonnegative function $[35,43]$,

(2) nonlocal approximations are used. 
To construct the numerical scheme for system (1) using NSFDM, the approximations of temporal derivatives are made based on generalized forward scheme of first order. Hence, if $g(t) \in C^{1}(\mathbb{R})$, we define its derivative as follows:

$$
\begin{aligned}
& \frac{d g(t)}{d t}=\frac{g(t+\Delta t)-g(t)}{\varphi(\triangle t)}+O(\varphi(\Delta t)), \\
& \text { as } \Delta t \longrightarrow 0,
\end{aligned}
$$

where $\varphi(\Delta t)$ is a real-valued function on $\mathbb{R}$ and $\Delta t=h$. In the following, the denominator functions are little complex functions of the step-size of time than the classical one [44].

\section{Conflicts of Interest}

The authors declare that there are no conflicts of interest regarding the publication of this paper.

\section{References}

[1] Global Tuberculosis Report 2014, Geneva, World Health Organization, 2014, http://www.who.int/tb/publications/global_report/en/.

[2] P. W. Uys, R. M. Warren, and P. D. van Helden, "A threshold value for the time delay to TB diagnosis," PLoS ONE, vol. 2, no. 8, article no. e757, 2007.

[3] C. T. Sreeramareddy, K. V. Panduru, J. Menten, and J. Van den Ende, "Time delays in diagnosis of pulmonary tuberculosis: a systematic review of literature," BMC Infectious Diseases, vol. 9, article 91, 2009.

[4] K. Toman, Tuberculosis Case-Finding and Chemotherapy: Questions and Answers, WHO, Geneva, Switzerland, 1979.

[5] C. Castillo-Chavez and Z. Feng, "To treat or not to treat: the case of tuberculosis," Journal of Mathematical Biology, vol. 35, no. 6, pp. 629-656, 1997.

[6] T. Cohen and M. Murray, "Modeling epidemics of multidrugresistant M. tuberculosis of heterogeneous fitness," Nature Medicine, vol. 10, no. 10, pp. 1117-1121, 2004.

[7] R. Denysiuk, C. J. Silva, and D. F. Torres, "Multiobjective approach to optimal control for a tuberculosis model," Optimization Methods \& Software, vol. 30, no. 5, pp. 893-910, 2015.

[8] P. M. Small and P. I. Fujiwara, "Management of tuberculosis in the United States," New England Journal of Medicine, vol. 345, no. 3, pp. 189-200, 2001.

[9] K. Styblo, "State of art: epidemiology of tuberculosis," Bulletin of the International Union against Tuberculosis, vol. 53, pp. 141-152, 1978.

[10] C. Silva, H. Maurer, and D. Torres, "Optimal control of a tuberculosis model with state and control delays," Mathematical Biosciences and Engineering. MBE, vol. 14, no. 1, pp. 321-337, 2017.

[11] F. A. Rihan and B. F. Rihan, "Numerical modelling of biological systems with memory using delay differential equations," Applied Mathematics and Information Sciences, vol. 9, no. 3, pp. 1615-1658, 2015.

[12] J. Arino and I. A. Soliman, "A model for the spread of tuberculosis with drug-sensitive and emerging multidrug-resistant and extensively drug-resistant strains," in Mathematical and Computational Modeling, pp. 1-120, Wiley, Hoboken, NJ, USA, 2015.
[13] N. H. Sweilam and S. M. Al-Mekhlafi, "Comparative study for multi-strain tuberculosis (TB) model of fractional order," Applied Mathematics and Information Sciences, vol. 10, no. 4, pp. 1403-1413, 2016.

[14] N. H. Sweilam, I. A. Soliman, and S. M. Al-Mekhlafi, "Nonstandard finite difference method for solving the multi-strain TB model," Journal of the Egyptian Mathematical Society, vol. 25, no. 2, pp. 129-138, 2017.

[15] N. H. Sweilam and S. M. AL-Mekhlafi, "On the optimal control for fractional multi-strain TB model," Optimal Control Applications \& Methods, vol. 37, no. 6, pp. 1355-1374, 2016.

[16] N. H. Sweilam and S. M. AL-Mekhlafi, "Numerical study for multi-strain tuberculosis (TB) model of variable-order fractional derivatives," Journal of Advanced Research, vol. 7, no. 2, pp. 271-283, 2016.

[17] A. M. Nagy and N. H. Sweilam, "An efficient method for solving fractional Hodgkin-HUXley model," Physics Letters. A, vol. 378, no. 30-31, pp. 1980-1984, 2014.

[18] N. H. Sweilam, M. M. Khader, and A. M. S. Mahdy, "Numerical studies for fractional-order logistic differential equation with two different delays," Journal of Applied Mathematics, vol. 2012, Article ID 764894, 2012.

[19] N. H. Sweilam, M. M. Khader, and M. Adel, "On the stability analysis of weighted average finite difference methods for fractional wave equation," Fractional Differential Calculus, vol. 2, no. 1, pp. 17-29, 2012.

[20] N. H. Sweilam, M. M. Khader, and A. M. Nagy, "Numerical solution of two-sided space-fractional wave equation using finite difference method," Journal of Computational and Applied Mathematics, vol. 235, no. 8, pp. 2832-2841, 2011.

[21] A. Bellen and M. Zennaro, Numerical Methods for Delay Differential Equations, Clarendon Press, Oxford, UK, 2003.

[22] R. Cooke and K. L. Bellman, Differential-Difference Equations, Academic Press, New York, NY, USA, 1963.

[23] R. Driver, Ordinary and Delay Differential Equations, Springer, Berlin, Germany, 1977.

[24] J. Hale, Theory of Functional Differential Equations, Springer, New York, NY, USA, 1977.

[25] V. G. Pimenov and E. E. Tashirova, "Numerical methods for solving a hereditary equation of hyperbolic type," Proceedings of the Steklov Institute of Mathematics, vol. 281, no. suppl. 1, pp. S126-S136, 2013.

[26] F. A. Rihan, A. Hashish, F. Al-Maskari, M. S. Hussein, E. Ahmed et al., "Dynamics of tumor-immune system with fractionalorder," Journal of Tumor Research, vol. 2, article 109, 2016.

[27] F. A. Rihan, S. Lakshmanan, A. H. Hashish, R. Rakkiyappan, and E. Ahmed, "Fractional-order delayed predator-prey systems with Holling type-II functional response," Nonlinear Dynamics. An International Journal of Nonlinear Dynamics and Chaos in Engineering Systems, vol. 80, no. 1-2, pp. 777-789, 2015.

[28] K. T. Alligood, T. D. Sauer, and J. A. Yorke, Chaos: An Introduction to Dynamical Systems, Springer, Berlin, Germany, 1997.

[29] V. Daftardar-Gejji, Y. Sukale, and S. Bhalekar, "Solving fractional delay differential equations: A new approach," Fractional Calculus and Applied Analysis, vol. 18, no. 2, pp. 400-418, 2015.

[30] L. C. Davis, "Modifications of the optimal velocity traffic model to include delay due to driver reaction time," Physica A: Statistical Mechanics and Its Applications, vol. 319, pp. 557-567, 2003.

[31] Y. Kuang, Delay Differential Equations with Applications in Population Biology, Academic Press, New York, NY, USA, 1993. 
[32] P. van den Driessche and J. Watmough, "Reproduction numbers and sub-threshold endemic equilibria for compartmental models of disease transmission," Mathematical Biosciences, vol. 180, pp. 29-48, 2002.

[33] Z. Wang, X. Huang, and J. Zhou, "A numerical method for delayed fractional-order differential equations: based on G-L definition," Applied Mathematics \& Information Sciences, vol. 7, no. 2L, pp. 525-529, 2013.

[34] F. A. Rihan, "Computational methods for delay parabolic and time-fractional partial differential equations," Numerical Methods for Partial Differential Equations. An International Journal, vol. 26, no. 6, pp. 1556-1571, 2010.

[35] R. E. Mickens, Nonstandard Finite Difference Models of Differential Equations, World Scientific, Singapore, 2005.

[36] R. Anguelov and J. M. Lubuma, "Nonstandard finite difference method by nonlocal approximation," Mathematics and Computers in Simulation, vol. 61, no. 3-6, pp. 465-475, 2003.

[37] A. J. Arenas, G. González-Parra, and B. M. Chen-Charpentier, "Construction of nonstandard finite difference schemes for the SI and SIR epidemic models of fractional order," Mathematics and Computers in Simulation, vol. 121, pp. 48-63, 2016.

[38] H. A. A. El-Saka, "The fractional-order SIR and SIRS epidemic models with variable population," Mathematical Sciences Letters, vol. 2, no. 3, pp. 195-200, 2013.

[39] D. Matignon, "Stability results for fractional differential equations with applications to control processing," in Computational Engineering in Systems and Application. in. Multiconference, IMACS, IEEE-SMC, vol. 2, pp. 963-968, Lille, France, 1996.

[40] K. S. Miller and B. Ross, An Introduction to the Fractional Calculus and Fractional Differential Equations, A Wiley-Interscience Publication, John Wiley \& Sons, New York, NY, USA, 1993.

[41] I. Podlubny, Fractional Differential Equations, Academic Press, New York, NY, USA, 1999.

[42] M. M. Meerschaert and C. Tadjeran, "Finite difference approximations for fractional advection-dispersion flow equations," Journal of Computational and Applied Mathematics, vol. 172, no. 1, pp. 65-77, 2004.

[43] R. E. Mickens, "Calculation of denominator functions for nonstandard finite difference schemes for differential equations satisfying a positivity condition," Numerical Methods for Partial Differential Equations. An International Journal, vol. 23, no. 3, pp. 672-691, 2007.

[44] H. A. Obaid, Construction and analysis of efficient numerical methods to solve Mathematical models of TB and HIV co-infection [Ph.D. thesis], University of the Western Cape, May 2011. 


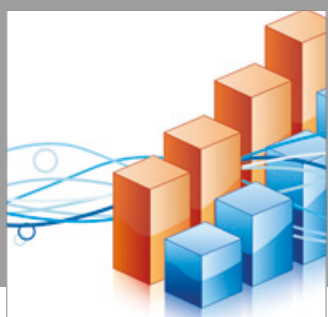

Advances in

Operations Research

vatersals

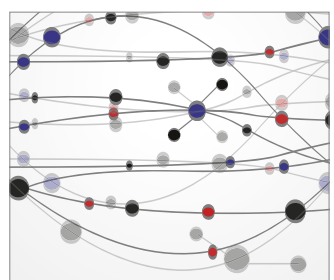

\section{The Scientific} World Journal
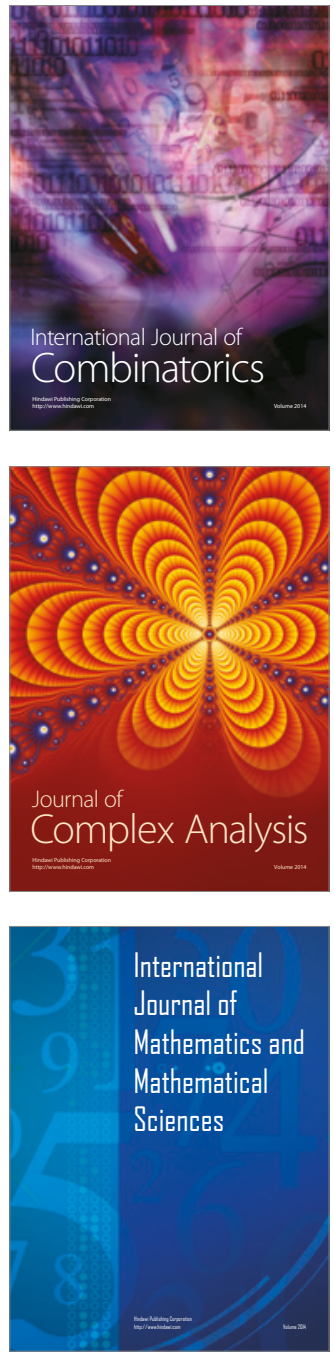
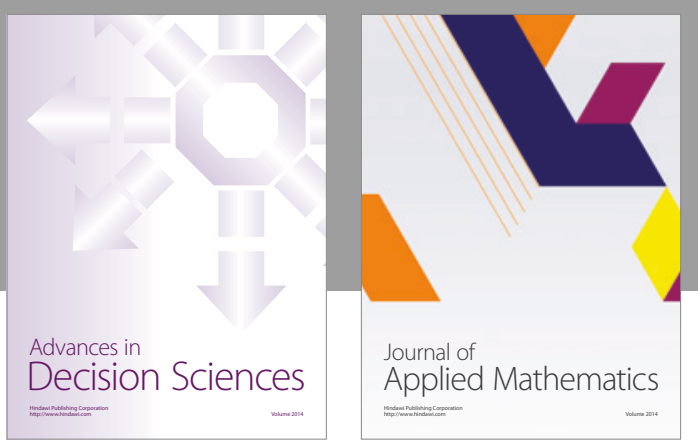

Algebra

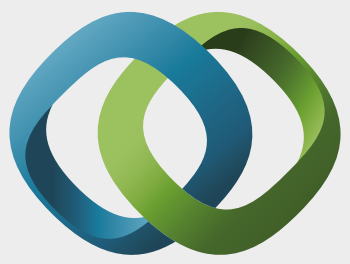

\section{Hindawi}

Submit your manuscripts at

https://www.hindawi.com
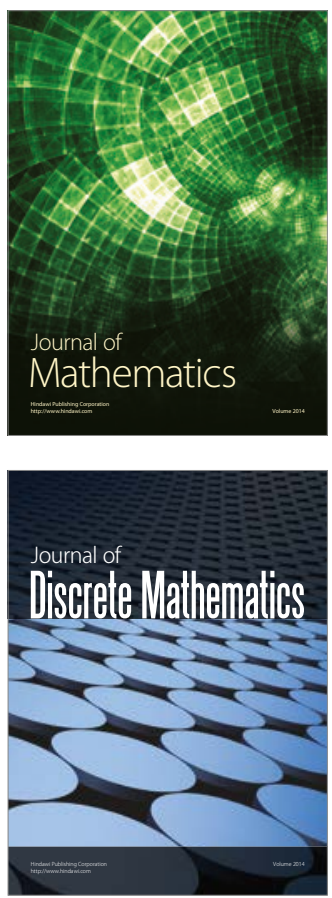

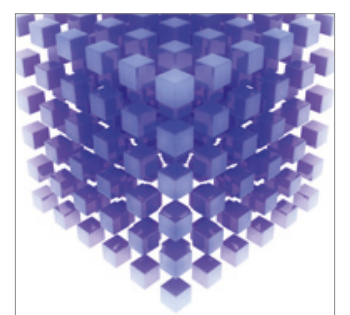

Mathematical Problems in Engineering
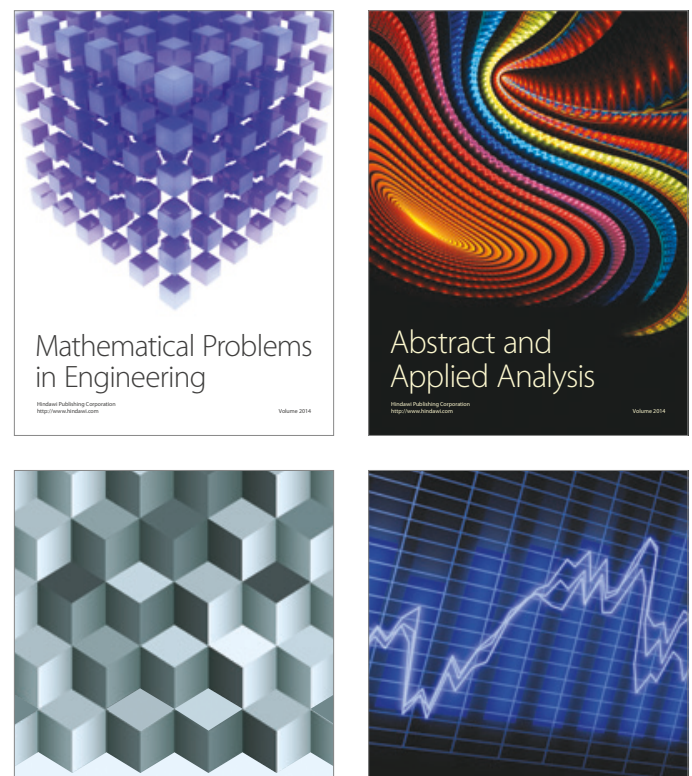

Journal of

Function Spaces

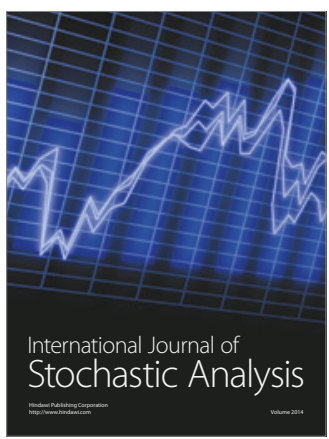

Probability and Statistics
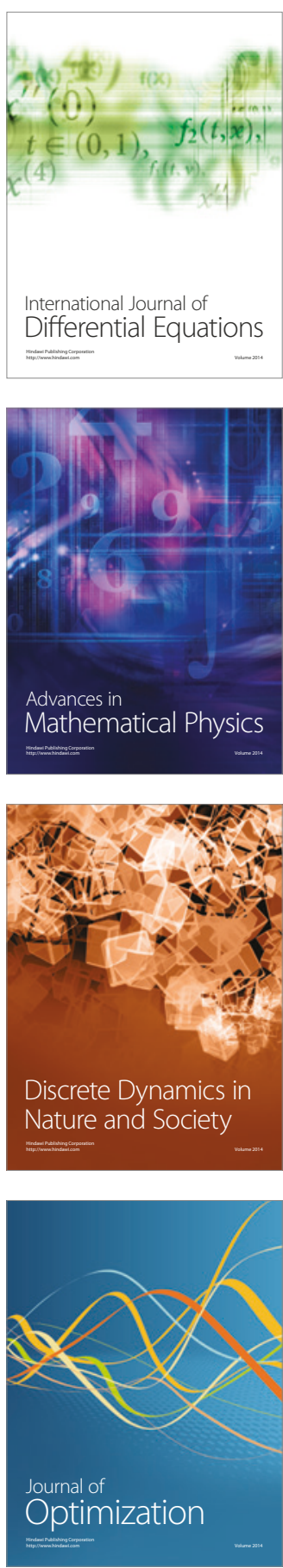